\title{
Isothermal Water Flows in Low Porosity Porous Media in Presence of Vapor-Liquid Phase Change
}

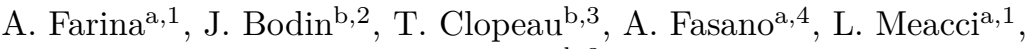 \\ A. Mikelićb,3,* \\ ${ }^{a}$ Dipartimento di Matematica "Ulisse Dini", Viale Morgagni 67/A, \\ I-50134 Firenze, ITALIA \\ ${ }^{b}$ Université de Lyon, Lyon, F-69003, FRANCE \\ Université Lyon 1, Institut Camille Jordan, \\ UMR 5208, Département de Mathématiques, 43 Bd du 11 novembre 1918, \\ 69622 Villeurbanne Cedex, FRANCE
}

\begin{abstract}
In this article we consider a mathematical model for a low porosity porous medium saturated by water, present both as the liquid and the vapor phase. In the isothermal case we propose a new formulation using a single nonlinear parabolic-hyperbolic equation for the fluid mixture density $X$. We present the derivation of the unified model and a number of numerical simulations based on regularization and Kirchhoff's transform.
\end{abstract}

Keywords: degenerate parabolic equation, non-saturated flows, entropy solutions, Kirchhoff's transform

\section{Introduction}

We study the flow of a water-vapor mixture saturating low porosity rocks. Such systems are present in many applications, particularly in geology. Assumption of low porosity allows to consider the situation in which the temperature field is constant, because it is stabilized by the dominating volume fraction of

\footnotetext{
*Corresponding author, Fax: +33956109885, Tel: +33426234548.

Email addresses: farina@math.unifi.it (A. Farina), jonathan.bodin@univ-lyon1.fr

(J. Bodin), thierry.clopeau@univ-lyon1.fr (T. Clopeau), fasano@math.unifi.it

(A. Fasano), andro.mikelic@univ-lyon1.fr (A. Mikelić)

${ }^{1}$ Research of A.F. and L.M. was partially supported by the project MAC-GEO, Regione Toscana.

${ }^{2}$ Ph.D. Student ANDRA, Parc de la Croix Blanche, 1-7 rue Jean Monnet, 92298 ChatneayMalabry CEDEX, FRANCE.

${ }^{3}$ Research of T.C. and A.M. was partially supported by the GNR MOMAS CNRS-2439 (Modélisation Mathématique et Simulations numériques liées aux problèmes de gestion des déchets nucléaires) (PACEN/CNRS, ANDRA, BRGM, CEA, EDF, IRSN).

${ }^{4}$ The research was partially supported by the Italian PRIN2008 Project "Mathematical Models for Multicomponent Systems in Medical and Environmental Sciences".
} 
the rocks. Therefore, the isothermal configuration can be justified even in the presence of phase change. In the classic approach, once the temperature is fixed, the state of the water is determined by the pressure. Under the assumption of thermodynamical equilibrium, by comparison with phase change pressure, corresponding to the given temperature, we find out whether the water is in the liquid or in the vapor state, or both phases are simultaneously present. Accordingly, mass balance is expressed by PDEs of different nature. The backdraw of such an approach is that it involves the explicit analysis of the interfaces, which is a hard task to achieve, especially in multidimensional problems.

There is a recent surge of interest for such questions in the case of multiphase multi-component flows through porous media. Contrary to case of two immiscible incompressible phases, for which there is an extensive literature (see e.g. [2], [14], [27] and references therein), the situation where we deal with at least one compressible phase remains with incomplete mathematical theory. For partial results we refer to [30]. In [21] constitutive laws for compressible phase were adapted to Chavent's global pressure.

One of the main difficulties is that in general we should deal with systems of degenerate parabolic PDEs and it is not clear how to guarantee a priori that saturations remain in the physical range. See [30] for remarks. Furthermore, there is appearance of single-phase zones occupied by the fluid which is over- (or under-) saturated. In an oversaturated zone the two-phase flow equations degenerate and cannot be longer used, which provokes serious numerical problems. Presence of several components clearly complicates the situation. Motivated by recent applied needs, Panfilov introduced new formulation, extending the concept of phase saturation so that it may be negative and larger than one. His method allowed using the existing numerical simulators of two-phase flow for modeling single-phase zones. For details we refer to [1] and [35]. A similar approach, but extending different unknowns, was presented by Bourgeat et al in $[9]$.

Our situation is much simpler, since we consider water as the only component and in isothermal conditions. Our aim is to describe the physical model simply by a scalar degenerate parabolic equation for the liquidvapor mixture density, a quantity possessing a natural definition. Of course it applies only to our particular situation, although some generalization may be possible (for instance to non-isothermal cases).

The plan of the paper is the following: In Section 2 we present derivation of the model. In the subsection 2.6 we define precisely the notion of the entropy solution and the regularization procedure, allowing us to define the numerical approximation. In Section 3, we present numerical simulations obtained by using the Comsol Multiphysics and the Scilab based numerical code.

\section{Mathematical Model}

The purpose of this section is to present a mathematical model including just one PDE for the liquid-vapor mixture density $X$. We will consider the two cases: absence or presence of capillarity. We simplified the model by considering 
just isothermal conditions, but extension to other temperature distributions are possible.

\subsection{Basic notations and assumptions}

We consider a porous medium saturated with pure $\mathrm{H}_{2} \mathrm{O}$, present both in the liquid phase and in the gaseous phase (vapor). The medium occupies some domain $\Omega \subset \mathbb{R}^{3}$ with smooth boundary.

So, from the macroscopic point of view, we deal with a mixture whose solid component is the porous skeleton and the fluid component is constituted by liquid and vapor. We thus denote by the suffix $l$ the liquid phase of the fluid and by the suffix $g$ the gaseous phase (vapor).

Let us list the main physical assumptions that we consider:

A1. The porous matrix is rigid and possibly non-homogeneous. So, if we denote by $\phi$ its porosity,

$$
\phi=\phi(\mathbf{x}), \quad \mathbf{x} \in \Omega .
$$

A2. There is no mass exchange between the solid matrix and the fluid.

A3. The pores are filled by $\mathrm{H}_{2} \mathrm{O}$, in either phase. Hence

$$
S_{l}+S_{g}=1,
$$

where $S_{\beta}, \beta=l, g$, denotes the pores volume fraction occupied by the corresponding phase.

A4. The porosity is so small that the amount of $\mathrm{H}_{2} \mathrm{O}$ present in either phase has no influence on the thermal field which is a priori prescribed. For the same reason we neglected the latent heat, possibly involved in the process. Therefore we suppose that locally there is thermal equilibrium between the phases and between them and the porous solid. Moreover, the temperature $T$ is uniform within the domain and constant in time. Clearly, this is not only a strong constraint, but it may also represent a severe physical limitation, since it requires that the latent heat absorbed or released, due to the phase change, is effectively dispersed by the solid matrix.

A5. The inertia of the fluid is negligible, i.e. the Reynolds number relative to the flow within the pores is $\mathcal{O}(1)$ or less. Moreover, we assume that Darcy's law governs the flux of both phases ${ }^{5}$.

A6. The gaseous phase is modeled as a perfect gas, but more complicated state equations can be considered.

A7. We introduce some mechanical compressibility of liquid water as a kind of regularization, which is helpful for numerical simulations.

\footnotetext{
${ }^{5}$ According to such an approach we neglect any mechanical dispersion phenomena.
} 
We denote by $P_{\beta}, \beta=l, g$, the $\mathrm{H}_{2} \mathrm{O}$ pressure in the $\beta$-th phase. Next, $\rho_{\beta}$, $\beta=l, g$, denotes the density of the $\beta$-th phase. In particular, by assumption A7, we have

$$
\rho_{l}=\rho_{l}^{o} e^{\alpha\left(P_{l}-P_{l}^{o}\right)},
$$

where $\rho_{l}^{o}, P_{l}^{o}$ are reference values and $\alpha$ is the isothermal mechanical compressibility factor (usually $\alpha\left(P_{l}-P_{l}^{o}\right) \ll 1$ ). From assumption $\mathbf{A 6}$ we derive

$$
\frac{P_{g}}{\rho_{g}}=\frac{\mathcal{R}}{\mathfrak{M}_{\mathrm{H}_{2} \mathrm{O}}} T=\mathfrak{r} T, \quad \text { where } \quad \mathfrak{r}=\frac{\mathcal{R}}{\mathfrak{M}_{\mathrm{H}_{2} \mathrm{O}}},
$$

with $\mathcal{R}$ perfect gas constant, $\mathcal{R}=8,314 \mathrm{~J} /\left(\mathrm{mol}^{\circ} \mathrm{K}\right)$, and $\mathfrak{M}_{\mathrm{H}_{2} \mathrm{O}}=18 \mathrm{~g} / \mathrm{mol}$, water molar mass. Hence $\mathfrak{r}=461,9 \mathrm{~J} /\left(\mathrm{kg}^{\circ} \mathrm{K}\right)$.

\subsection{Momentum balance}

Accordingly to the assumption A5, we will write for the liquid phase

$$
\mathbf{q}_{l}=-\mathbb{K} \frac{k_{r, l}\left(S_{l}\right)}{\mu_{l}}\left(\nabla P_{l}-\rho_{l} \mathbf{g}\right),
$$

and for the vapor phase

$$
\mathbf{q}_{g}=-\mathbb{K} \frac{k_{r, g}\left(S_{g}\right)}{\mu_{g}}\left(\nabla P_{g}-\rho_{g} \mathbf{g}\right),
$$

where:

- $\mathbf{q}_{\beta}, \beta=l, g$, is the $\beta$-th phase volumetric macroscopic discharge within the porous medium. Hence $\rho_{\beta} \mathbf{q}_{\beta}$ is the corresponding mass flux.

- $\mathrm{g}$ is the gravity acceleration.

- $\mu_{\beta}, \beta=l, g$, is the viscosity of the $\beta$-th phase.

- $\mathbb{K}$ is the intrinsic medium permeability tensor $\left[L^{2}\right]$. We allow $\mathbb{K}$ to depend on $\mathbf{x}$, but not on time.

- $k_{r, \beta}\left(S_{\beta}\right), \beta=l, g$, is the relative permeability of the $\beta$-th phase.

We suppose that all the functions introduced above are smooth. Moreover, we remark that in writing (4) and (5) we have implicitly assumed that the interaction between liquid and vapor is negligible with respect to the one with the solid matrix. Such an assumption is reasonable when the vapor-liquid relative velocity is small compared with the phases velocities, i.e. $\left|\mathbf{q}_{l}-\mathbf{q}_{g}\right| \ll\left|\mathbf{q}_{g}\right|$, and $\left|\mathbf{q}_{l}-\mathbf{q}_{g}\right| \ll\left|\mathbf{q}_{l}\right|$.

We denote by $P$ the $\mathrm{H}_{2} \mathrm{O}$ pressure. We have

$$
\begin{array}{lll}
S_{l}=1 & \Leftrightarrow & P_{l}=P, \\
S_{l}=0 & \Leftrightarrow & P_{g}=P .
\end{array}
$$


When $0<S_{l}<1, P_{l}$ and $P_{g}$ need to be specified constitutively. In such a case we treat the fluid (namely liquid and vapor) as a mixture and then $P$ represents the pressure of the mixture itself identifiable with the pressure of phase equilibrium.

Finally, we denote by $\hat{\rho}_{\beta}$ the mass of $\beta$-th phase per unit volume of porous medium. Hence (see for instance [40])

$$
\hat{\rho}_{\beta}=\phi S_{\beta} \rho_{\beta}, \quad \text { with } \beta=l, g .
$$

\subsection{Mass balance}

Let us write down the global mass balance equation

$$
\frac{\partial \hat{\rho}}{\partial t}+\nabla \cdot\left(\rho_{l} \mathbf{q}_{l}+\rho_{g} \mathbf{q}_{g}\right)=0
$$

where $^{6}$ :

- $\hat{\rho}=\hat{\rho}_{l}+\hat{\rho}_{g}=\phi\left(S \rho_{l}+(1-S) \rho_{g}\right)$, is the total mass of $\mathrm{H}_{2} \mathrm{O}$ per unit volume of porous medium.

- $\left(\rho_{l} \mathbf{q}_{l}+\rho_{g} \mathbf{q}_{g}\right)$, is the total water flux. Hence, recalling (4) and (5)

$$
\rho_{l} \mathbf{q}_{l}+\rho_{g} \mathbf{q}_{g}=-\mathbb{K}\left[\rho_{l} \frac{k_{r, l}(S)}{\mu_{l}}\left(\nabla P_{l}-\rho_{l} \mathbf{g}\right)+\rho_{g} \frac{k_{r, g}(1-S)}{\mu_{g}}\left(\nabla P_{g}-\rho_{g} \mathbf{g}\right)\right] .
$$

Using (1)-(7), we are in position to write the $\mathrm{H}_{2} \mathrm{O}$ mass balance equation

$$
\begin{aligned}
& \phi \frac{\partial}{\partial t}\left[S \rho_{l}+\rho_{g}(1-S)\right]-\nabla \cdot\left[\mathbb { K } \left(\rho_{l} \frac{k_{r, l}(S)}{\mu_{l}}\left(\nabla P_{l}-\rho_{l} \mathbf{g}\right)\right.\right. \\
& \left.\left.+\rho_{g} \frac{k_{r, g}(1-S)}{\mu_{g}}\left(\nabla P_{g}-\rho_{g} \mathbf{g}\right)\right)\right]=0,
\end{aligned}
$$

with $\rho_{g}$ given in terms of $P_{g}$ by (3) and, according to A 7, $\rho_{l}$ expressed in terms of $P_{l}$ by $(2)$.

Before proceeding further, some comments relative to (8) are in order. First, if only the liquid phase is present, $S=1$, then (8) reduces to

$$
\phi \frac{\partial \rho_{l}}{\partial t}=\nabla \cdot\left[\frac{\mathbb{K} \rho_{l}}{\mu_{l}}\left(\nabla P_{l}-\rho_{l} \mathbf{g}\right)\right],
$$

which can be read as a parabolic equation whose unknown is $P_{l}$ that, of course, coincides with $P$

$$
\phi \alpha \rho_{l} \frac{\partial P_{l}}{\partial t}=\nabla \cdot\left[\frac{\mathbb{K} \rho_{l}\left(P_{l}\right)}{\mu_{l}}\left(\nabla P_{l}-\rho_{l} \mathbf{g}\right)\right]
$$

\footnotetext{
${ }^{6}$ For simplicity, here and in the sequel we put $S_{l}=S$, and $S_{g}=1-S$.
} 
Equation (10) is the classical porous media equation when the fluid mechanical compressibility is taken into account [8]. Once solved, with suitable boundary conditions, equation (10) gives the pressure field in the liquid phase and, from

(4) it allows to evaluate the liquid flux. We finally remark that, by virtue of (2), equation (10) can also be written in terms of $\rho_{l}$, getting

$$
\phi \frac{\partial \rho_{l}}{\partial t}=\nabla \cdot\left[\frac{\mathbb{K}}{\mu_{l}}\left(\frac{1}{\alpha} \nabla \rho_{l}-\rho_{l}^{2} \mathbf{g}\right)\right] .
$$

In any case we end up with a parabolic equation.

Let us now consider $S=0$. Equation (8) is then

$$
\frac{\partial}{\partial t}\left(\phi \rho_{g}\right)+\nabla \cdot\left(\rho_{g} \mathbf{q}_{g}\right)=0, \Rightarrow \phi \frac{\partial P_{g}}{\partial t}-\nabla \cdot\left[\frac{P_{g}}{\mu_{g}} \mathbb{K}\left(\nabla P_{g}-\frac{P_{g}}{\mathfrak{r} T} \mathbf{g}\right)\right]=0
$$

where we have used (3) for expressing $\rho_{g}$ in terms of $P_{g}$. Of course, one could have also selected $\rho_{g}$ as the primary variable, obtaining

$$
\phi \frac{\partial \rho_{g}}{\partial t}-\nabla \cdot\left[\frac{\rho_{g}}{\mu_{g}} \mathbb{K}\left(\mathfrak{r} T \nabla \rho_{g}-\rho_{g} \mathbf{g}\right)\right]=0 .
$$

When liquid and vapor are simultaneously present within the porous medium, the unknowns are : $S, P_{l}$ and $P_{g}$, and we need a constitutive relation to complete the model. This issue will be analyzed in the following sections.

\subsection{Mathematical model in absence of capillarity}

The Clapeyron diagram in the $(P, T)$ plane provides the phase change curves, separating the different phases. In particular, the so-called saturation pressure curve or boiling pressure curve ${ }^{7}$

$$
P=P^{*}(T),
$$

separates liquid and vapor phases. Hence, for fixed $T$, if $P>P^{*}$, then the $\mathrm{H}_{2} \mathrm{O}$ is in the liquid phase $\left(P_{l}=P\right)$. Conversely if $P<P^{*}$ then the $\mathrm{H}_{2} \mathrm{O}$ is the gaseous phase $\left(P_{g}=P\right)$.

When liquid and vapor coexist, $P_{l}$ and $P_{g}$ have to be specified constitutively.

If capillary effects are neglected ${ }^{8}$, the simplest constitutive assumption (often used in the literature, see [20] for instance) is the following

$$
P_{g}=P_{l}=P^{*}
$$

\footnotetext{
${ }^{7}$ For pressure in the range $10^{5}-10^{7} \mathrm{~Pa}$, a good approximation of $P^{*}$ is the following [42],$$
P^{*}(T)=P_{a} \exp \left\{A+\frac{B}{T}\right\},
$$

with $P_{a}=10^{5} \mathrm{~Pa}, A=12,512, B=-4611,73^{\circ} \mathrm{K}$.

${ }^{8}$ Capillarity will be dealt with in the next section.
} 
In such a case the primary variable becomes the liquid saturation $S$. Concerning the densities, we introduce $\rho_{l}^{*}$ and $\rho_{g}^{*}$, setting

$$
\begin{gathered}
\rho_{l}^{*}=\rho_{l}\left(P_{l}=P^{*}\right)=\rho_{l}^{o} \exp \left\{\alpha\left(P^{*}-P_{l}^{o}\right)\right\}, \\
\rho_{g}^{*}=\rho_{g}\left(P_{g}=P^{*}\right)=\frac{P^{*}(T)}{\mathfrak{r} T} .
\end{gathered}
$$

Let us now denote by $X$ the global density of the liquid-vapor mixture, i.e. the mass of $\mathrm{H}_{2} \mathrm{O}$ per unit volume occupied by water, irrespectively of the phase. When $0<S<1$,

$$
X=S \rho_{l}^{*}+(1-S) \rho_{g}^{*}
$$

while

$$
\begin{aligned}
& X=\rho_{l}, \quad\left(\text { and } X \geqslant \rho_{l}^{*}\right) \text { when } S=1, \\
& X=\rho_{g}, \quad\left(\text { and } X \leqslant \rho_{g}^{*}\right) \text { when } S=0 .
\end{aligned}
$$

Next, we observe that

$$
\frac{d X}{d S}>0, \text { when } 0<S<1,
$$

provided $\rho_{l}^{*}>\rho_{g}^{*}$, (always fulfilled if we are far from the critical point). So

$$
\rho_{g}^{*}=X(0)<X(S)<X(1)=\rho_{l}^{*}, \text { for } S \in(0,1) .
$$

We thus may express $S$ in terms of $X$ defining the following function

$$
S(X)= \begin{cases}0, & \text { if } \quad X \leqslant X(0)=\rho_{g}^{*}, \\ \frac{X-\rho_{g}^{*}}{\rho_{l}^{*}-\rho_{g}^{*}}, & \text { if } \rho_{g}^{*}<X<\rho_{l}^{*}, \\ 1 & \text { if } X \geqslant X(1)=\rho_{l}^{*} .\end{cases}
$$

Hence, recalling (2) and (3), we may give such general constitutive relations between $P_{\beta}, \beta=l, g$, and $X$,

$$
\begin{gathered}
P_{l}(X)=\left\{\begin{array}{lr}
\frac{1}{\alpha} \ln \left(\frac{X}{\rho_{l}^{o}}\right)+P_{l}^{o}, & \text { if } X>X(1)=\rho_{l}^{*}, \\
P^{*}, & \text { if } X \leqslant X(1)=\rho_{l}^{*},
\end{array}\right. \\
P_{g}(X)= \begin{cases}P^{*}, & \text { if } X>X(0)=\rho_{g}^{*}, \\
\mathfrak{r} T X, & \text { if } X \leqslant X(0)=\rho_{g}^{*} .\end{cases}
\end{gathered}
$$


So, when capillary effects can be neglected, the $\mathrm{H}_{2} \mathrm{O}$ flow within the porous medium is governed by the following equation

$$
\phi \frac{\partial X}{\partial t}-\nabla \cdot[\mathbb{F}(X, \mathbf{x}) \nabla X+\mathbf{G}(X, \mathbf{x})]=0
$$

where:

- If $X \geqslant \rho_{l}^{*}$,

$$
\left\{\begin{array}{l}
\mathbb{F}(X, \mathbf{x})=\frac{1}{\alpha \mu_{l}} \mathbb{K}(\mathbf{x}), \\
\mathbf{G}(X, \mathbf{x})=-\frac{X^{2}}{\mu_{l}} \mathbb{K}(\mathbf{x}) \mathbf{g} .
\end{array}\right.
$$

- If $\rho_{g}^{*}<X<\rho_{l}^{*}$,

$$
\left\{\begin{array}{l}
\mathbb{F}(X, \mathbf{x})=0 \\
\mathbf{G}(X, \mathbf{x})=-\left(\rho_{l}^{* 2} \frac{k_{r, l}(S(X))}{\mu_{l}}+\rho_{g}^{* 2} \frac{k_{r, g}(1-S(X))}{\mu_{g}}\right) \mathbb{K}(\mathbf{x}) \mathbf{g} .
\end{array}\right.
$$

- If $X \leqslant \rho_{g}^{*}$,

$$
\left\{\begin{array}{l}
\mathbb{F}(X, \mathbf{x})=\frac{X \mathfrak{r} T}{\mu_{g}} \mathbb{K}(\mathbf{x}) \\
\mathbf{G}(X, \mathbf{x})=-\frac{X^{2}}{\mu_{g}} \mathbb{K}(\mathbf{x}) \mathbf{g} .
\end{array}\right.
$$
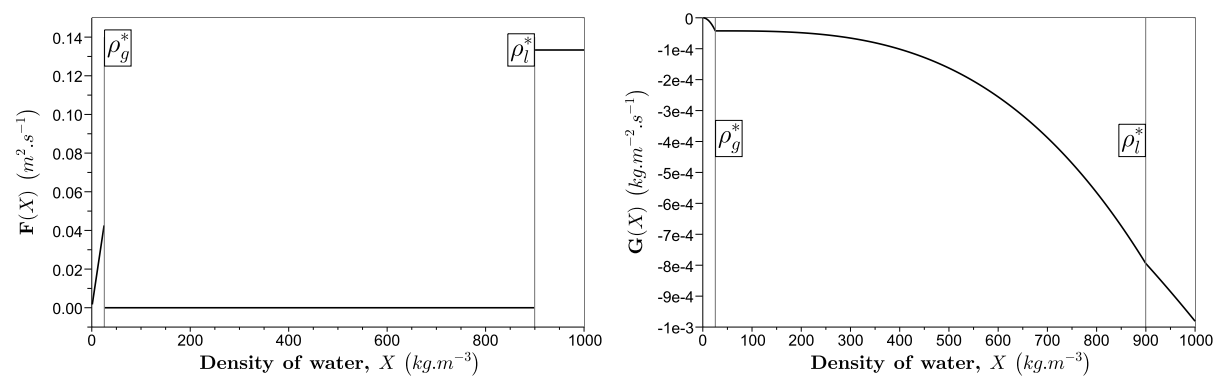

Figure 1: Qualitative behavior of $F(X)$ and $G(X)$, where $K=10^{-13} \mathrm{~m}^{2}, T=553^{\circ} \mathrm{K}$, $g=9.81 \mathrm{~m} . \mathrm{s}^{-2}, \alpha=7,5.10^{-10} \mathrm{~Pa}^{-1}, P_{l}^{o}=7 \mathrm{MPa}, \rho_{l}^{o}=900 \mathrm{~kg} \cdot \mathrm{m}^{-3}, \mu_{l}=10^{-3}$ Pa.s, $\mu_{g}=1,5.10^{-5}$ Pa.s, $k_{r, l}(S)=S^{3}$ and $k_{r, g}(1-S)=1-S^{3}$. Such values of parameters are typical of geothermal basins at the depth of $2-3 \mathrm{~km}$.

The qualitative behavior of $F(X)$ and $G(X)$ is shown in Figure 1. We remark that, when $\rho_{g}^{*}<X<\rho_{l}^{*}$, (20) is a hyperbolic equation. Indeed, in the presence 
of mixed phases and neglecting (according to (13)) capillary effects, pressure is just a function of temperature (which in our case is uniform and constant).

In the other cases, i.e. $X \leqslant \rho_{g}^{*}$, and $X \geqslant \rho_{l}^{*},(20)$ is parabolic and reduces to $(12)$ or to $(11)$.

\subsection{Mathematical model when capillary effects are non-negligible.}

If capillary phenomena are non-negligible we introduce (when liquid and vapor coexist) the so-called capillary pressure ${ }^{9}$

$$
P_{c}=P_{g}-P_{l}, \quad \Leftrightarrow \quad P_{l}=P_{g}-P_{c},
$$

with $P_{c}$ given by the well known Laplace formula

$$
P_{c}=2 \gamma\langle\kappa\rangle
$$

where:

- $\gamma=\gamma(T)$ surface tension (which in our case is constant).

- $\langle\kappa\rangle$ mean curvature of the vapor-liquid interface within the REV.

Equation (23) replaces (13). Next, we assume:

A8. The vapor pressure is given by

$$
P_{g}=P^{*} \exp \left\{-\frac{P_{c}}{\mathfrak{r} T \rho_{l}^{o}}\right\},
$$

i.e. by the the well known Kelvin's formula [16].

Formula (23) becomes

$$
P_{l}=-P_{c}+P^{*} \exp \left\{-\frac{P_{c}}{\mathfrak{r} T \rho_{l}^{o}}\right\} .
$$

Having introduced the additional unknown $P_{c}$, we need a constitutive relation linking it to some other variables. According to classical literature (see [7] and [8], for instance), we assume:

A9. The capillary pressure $P_{c}$ is an experimentally known function of the liquid saturation $S$, i.e.

$$
P_{c}=\widehat{P}_{c}(S),
$$

somehow expressing a relationship between $\langle\kappa\rangle$ and $S$. The function $\widehat{P}_{c}(S)$ is usually referred to as saturation curve.

\footnotetext{
${ }^{9}$ Since the liquid phase is the wetting phase $P_{g} \geqslant P_{l}$, entailing $P_{c} \geqslant 0$.
} 
We make some hypotheses on $\widehat{P}_{c}$. We start observing that, when $S$ is small, i.e. when the capillarity is expected to be important, $P_{g}-P_{l}$ must approach its maximum. On the other hand, when $S \rightarrow 1$, i.e. when capillarity phenomena become negligible, $P_{g}$ and $P_{l}$ must both tend to $P^{*}$, in agreement with (13). We thus suppose that $\widehat{P}_{c}(S)$ is bounded and non increasing, namely

$$
0 \leq P_{c}^{m}<\widehat{P}_{c}(S)<P_{c}^{M} \text {, for } 0<S<1 \text {, with } P_{c}^{m}=\widehat{P}_{c}(1), P_{c}^{M}=\widehat{P}_{c}(0),
$$

and

$$
\frac{d \widehat{P}_{c}(S)}{d S} \leqslant 0, \quad 0 \leqslant S \leqslant 1 .
$$

The lower and upper bound of $\widehat{P}_{c}(S)$, i.e. $P_{c}^{m}$ and $P_{c}^{M}$, depend, in general, on the particular structure of the porous matrix. The lower bound $P_{c}^{m}$ (usually referred to as entry pressure) and is very small, so that in many cases can be considered 0 .

We remark that the presence of capillarity diminishes the vapor pressure $P_{g}$. However the amount of decrease of the vapor pressure curve in a geothermal reservoir, is not completely understood [20].

Next, exploiting (3) and (25), we define

$$
\begin{aligned}
\rho_{g}(S) & =\frac{P^{*}}{\mathfrak{r} T} \exp \left\{-\frac{\widehat{P}_{c}(S)}{\mathfrak{r} T \rho_{l}^{o}}\right\} \\
& =\rho_{g}^{*} \exp \left\{-\frac{\widehat{P}_{c}(S)}{\mathfrak{r} T \rho_{l}^{o}}\right\},
\end{aligned}
$$

with $\rho_{g}^{*}$ given by (15), and, exploiting (2) and (26),

$$
\begin{aligned}
\rho_{l}(S) & =\rho_{l}^{o} \exp \left\{\alpha\left[-\widehat{P}_{c}(S)+P^{*} \exp \left\{-\frac{\widehat{P}_{c}(S)}{\mathfrak{r} T \rho_{l}^{o}}\right\}-P_{l}^{o}\right]\right\} \\
& =\rho_{l}^{*} \exp \left\{-\alpha\left[\widehat{P}_{c}(S)+P^{*}\left(1-\exp \left\{-\frac{\widehat{P}_{c}(S)}{\mathfrak{r} T \rho_{l}^{o}}\right\}\right)\right]\right\},
\end{aligned}
$$

with $\rho_{l}^{*}$ given by (14). We then introduce

$$
\rho_{g, c}^{*}=\lim _{S \rightarrow 0^{+}} \rho_{g}(S), \quad \text { and } \quad \rho_{l, c}^{*}=\lim _{S \rightarrow 1^{-}} \rho_{l}(S) .
$$

Thus, recalling (27), by (29), we have

$$
\rho_{g, c}^{*}=\lim _{S \rightarrow 0^{+}} \rho_{g}(S)=\rho_{g}^{*} \exp \left\{-\frac{P_{c}^{M}}{\mathfrak{r} T \rho_{l}^{o}}\right\},
$$

and by (30),

$$
\rho_{l, c}^{*}=\lim _{S \rightarrow 1^{-}} \rho_{l}(S)=\rho_{l}^{*} \exp \left\{-\alpha\left[P_{c}^{m}+P^{*}\left(1-\exp \left\{-\frac{P_{c}^{m}}{\mathfrak{r} T \rho_{l}^{o}}\right\}\right)\right]\right\},
$$


which, in case $P_{c}^{m}=0$, gives $\rho_{l, c}^{*}=\rho_{l}^{*}$, as physically expected. It is simple to prove that in the unsaturated zone $(0<S<1)$, the densities $\rho_{g}(S)$ and $\rho_{l}(S)$ are bounded both above and below

$$
\begin{gathered}
0<\rho_{g, c}^{*} \leqslant \rho_{g}(S) \leqslant \rho_{g}^{*}, \\
0<\rho_{l}^{o} e^{-\alpha\left(P_{c}^{M}+P_{l}^{o}\right)} \leqslant \rho_{l}(S) \leqslant \rho_{l, c}^{*} \leqslant \rho_{l}^{*} .
\end{gathered}
$$

So, when capillarity is not neglected, exploiting (25), (26), (2), and (3) we have

$$
\begin{aligned}
& P_{l}= \begin{cases}\frac{1}{\alpha} \ln \left(\frac{\rho_{l}}{\rho_{l}^{o}}\right)+P_{l}^{o}, & \text { if } \rho_{l} \geqslant \rho_{l, c}^{*}, \\
-\widehat{P}_{c}(S)+P^{*} \exp \left\{-\frac{\widehat{P}_{c}(S)}{\left.\mathfrak{r} T \rho_{l}^{o}\right\},}\right. & \text { if } \rho_{l}<\rho_{l, c}^{*},\end{cases} \\
& P_{g}= \begin{cases}\rho_{g} \mathfrak{r} T, & \text { if } \rho_{g} \leqslant \rho_{g, c}^{*}, \\
P^{*} \exp \left\{-\frac{\widehat{P}_{c}(S)}{\left.\mathfrak{r} T \rho_{l}^{o}\right\},}\right. & \text { if } \rho_{g}>\rho_{g, c}^{*} .\end{cases}
\end{aligned}
$$

Recalling now the definition of the global water density (16), we have

$$
X=S \rho_{l}(S)+(1-S) \rho_{g}(S),
$$

with $\rho_{g}(S), \rho_{l}(S)$ given by (29) and (30), respectively. We require, for physical coherence, that $X(S)$ is strictly increasing for $0<S<1$. By using (33) and $(34)$

$$
\begin{aligned}
\frac{\partial X}{\partial S} & =\rho_{l}(S)-\rho_{g}(S)+S \frac{\partial \rho_{l}(S)}{\partial S}+(1-S) \frac{\partial \rho_{g}(S)}{\partial S}, \\
& \geqslant \rho_{l}(S)-\rho_{g}(S), \\
& \geqslant \rho_{l}^{o} e^{-\alpha\left(P_{c}^{M}+P_{l}^{o}\right)}-\rho_{g}^{*} .
\end{aligned}
$$

Therefore it is enough that this lower bound function is strictly positive. Its behavior depends on the estimate for the maximum value of the capillary function $P_{c}^{M}$ satisfying

$$
P_{c}^{M}<\frac{1}{\alpha} \ln \left(\frac{\rho_{l}^{o}}{\rho_{g}^{*}}\right)-P_{l}^{o}
$$

Now using $\alpha P^{*} \ll 1$ we see that $X(S)$ is strictly increasing for $0<S<1$. So,

$$
X(0)<X(S)<X(1), \quad \text { for } \quad S \in(0,1),
$$

where now $X(0)=\rho_{g, c}^{*}, \quad X(1)=\rho_{l, c}^{*}$, with $\rho_{g, c}^{*}, \rho_{l, c}^{*}$ given by $(31),(32)$, respectively. 
Now, proceeding as in section 2.4, we express $S$ in terms of $X$ setting ${ }^{10}$

$$
S(X)= \begin{cases}0, & \text { if } X \leqslant \rho_{g, c}^{*}, \\ \text { inverse of }(37), & \text { if } \rho_{g, c}^{*}<X<\rho_{l, c}^{*}, \\ 1, & \text { if } X \geqslant \rho_{l, c}^{*} .\end{cases}
$$

So, remembering (35) and (36), we have

$$
\begin{gathered}
P_{l}(X)= \begin{cases}\frac{1}{\alpha} \ln \left(\frac{X}{\rho_{l}^{o}}\right)+P_{l}^{o}, & \text { if } X \geqslant \rho_{l, c}^{*}, \\
-\widehat{P}_{c}(S(X))+P^{*} \exp \left\{-\frac{\widehat{P}_{c}(S(X))}{\mathfrak{r} T \rho_{l}^{o}}\right\}, & \text { if } X<\rho_{l, c}^{*},\end{cases} \\
P_{g}(X)= \begin{cases}P^{*} \exp \left\{-\frac{\widehat{P}_{c}(S(X))}{\left.\mathfrak{r} T \rho_{l}^{o}\right\},}\right. & \text { if } X>\rho_{g, c}^{*}, \\
\mathfrak{r} T X, & \text { if } X \leqslant \rho_{g, c}^{*},\end{cases}
\end{gathered}
$$

which replace (18) and (19), respectively.

We thus end up again with equation (20), where now:

- If $X \leqslant \rho_{g, c}^{*}$, or $X \geqslant \rho_{l, c}^{*},(22)$ and (21) hold true, respectively.

- If $\rho_{g, c}^{*}<X<\rho_{l, c}^{*}$,

$$
\begin{aligned}
\mathbb{F}(X, \mathbf{x})= & {\left[\frac{\rho_{l}(S(X))}{\mu_{l}} k_{r, l}(S(X)) \frac{\partial P_{l}}{\partial S}\right.} \\
& \left.+\frac{\rho_{g}(S(X))}{\mu_{g}} k_{r, g}(1-S(X)) \frac{\partial P_{g}}{\partial S}\right] \frac{\partial S}{\partial X} \mathbb{K}(\mathbf{x}) \\
\mathbf{G}(X, \mathbf{x})=- & {\left[\frac{\left(\rho_{l}(S(X))\right)^{2}}{\mu_{l}} k_{r, l}(S(X))\right.} \\
& \left.+\frac{\left(\rho_{g}(S(X))\right)^{2}}{\mu_{g}} k_{r, g}(1-S(X))\right] \mathbb{K}(\mathbf{x}) \mathbf{g} .
\end{aligned}
$$

Note that now, when $\rho_{g, c}^{*}<X<\rho_{l, c}^{*},(20)$ is a parabolic equation.

\footnotetext{
${ }^{10}$ The inversion of (37) has to be performed numerically.
} 


\subsection{Some comments on the mathematical model}

For the model where the capillary effects are taken into account, we will consider two families of functions for $\widehat{P}_{c}, k_{r, l}$ and $k_{r, g}$ (see Table 1). In the first case the capillary pressure function is a linear function and the relative permeability functions a cubic function of the saturation. In the second case the capillary pressure function is given by the van Genuchten model (see [23]) and relative permeability functions by the van Genuchten-Mualem model (see [32] and [23]).

\begin{tabular}{cccc} 
& $\widehat{P}_{c}(S)$ & $k_{r, l}$ & $k_{r, g}$ \\
\hline Linear & $P_{c}^{M}(1-S)$ & $S^{3}$ & $1-S^{3}$ \\
Van G. M. & $P_{r}\left[(S)^{-1 / m}-1\right]^{1 / n}$ & $\sqrt{S}\left[1-\left(1-S^{1 / m}\right)^{m}\right]^{2}$ & $1-k_{r, l}(S)$ \\
\hline
\end{tabular}

Table 1: Families of capillary and relative permeability functions. $n=6.6$ and $m=1-1 / n$.

When the van Genuchten model is considered, derivative of $\widehat{P}_{c}$, has singularities at $S=0$ and $S=1$. Therefore, we regularize this function in a small neighborhood of 0 and 1 by a quadratic function. In particular, we impose the continuity of $\widehat{P}_{c}$ and of its derivatives at $S=\varepsilon$ and $S=1-\varepsilon\left(\varepsilon=10^{-4}\right.$ for example), and we close the system by imposing $\widehat{P}_{c}(0)=P_{c}^{M}$ and $\widehat{P}_{c}(1)=0$.

We now assume that $\mathbb{K}=K \mathbb{I}$, with $K$ uniform so that

$$
\mathbb{F}(X)=F(X) \mathbb{I} .
$$

Then using the results from the previous subsection, we have

H1 G is locally Lipschitz continuous.

H2 $\int_{0}^{s} F(\eta) d \eta$ is a nondecreasing Lipschitz continuous function.

Since, the equation (20) changes the type, we should define precisely the notion of the variational solution.

A fairly complete theory of the degenerate parabolic equations on a bounded domain is in the paper [29], generalizing the seminal paper of Carrillo [13]. We consider the equation (20) in a smooth bounded domain $\Omega \subset \mathbb{R}^{n}$, with the following boundary and initial conditions

$$
\left.X\right|_{\partial \Omega}=X_{D},\left.\quad X\right|_{t=0}=X_{0} .
$$

with

H3 $X_{0} \in L^{\infty}(\Omega)$.

H4 $X_{D} \in L^{\infty}((0, T) \times \partial \Omega)$ and is a trace of a function $X_{D} \in L^{\infty}((0, T) \times \Omega)$ with $\int_{0}^{X_{D}} F(\eta) d \eta \in L^{2}\left(0, T ; H^{1}(\Omega)\right)$. 
H5 Let $B$ be a cover for $\bar{\Omega}$ such that, after a change of coordinates, the set $\Omega \cap B$ is epigraph of a $C^{1,1}$-function representing the boundary. We suppose $X_{D} \in W^{1,1}((0, T) \times B \cap Q)$ and $\Delta X_{D} \in L^{1}((0, T) \times B \cap Q)$, for every such cover $B$.

H6 $A=\min \left\{\inf _{\Omega} X_{0}, \inf _{\Omega \times(0, T)} X_{D}\right\}, \quad B=\max \left\{\sup _{\Omega} X_{0}, \sup _{\Omega \times(0, T)} X_{D}\right\}$ and $M=$ $\max _{s \in[A, B]}\left|\frac{d \mathbf{G}}{d X}(s)\right|$.

Then we set

Definition 1. (see [29], page 2265) A function $X \in L^{\infty}((0, T) \times \Omega)$ is said to be an entropy weak solution to problem (20), (44), that is, if $\int_{X_{D}}^{X} F(\eta) d \eta \in$ $L^{2}\left(0, T ; H_{0}^{1}(\Omega)\right)$ and for every $\theta \in C_{0}^{\infty}([0, T) \times \Omega)$,

$\int_{0}^{T} \int_{\Omega}\left(\phi X \partial_{t} \theta+\left(\mathbf{G}(X)-\nabla \int_{0}^{X} F(\eta) d \eta\right) \cdot \nabla \theta\right) d x d t+\int_{\Omega} X_{0} \theta(0, x) d x=0$

and if it satisfies the following entropy inequalities for all $s \in[A, B]$, for all $\psi \in C_{0}^{\infty}\left([0, T) \times \mathbb{R}^{n}\right)$ such that $\psi \geq 0$ and $\operatorname{sign}^{ \pm}\left(\int_{X_{D}}^{X} F(\eta) d \eta\right) \psi=0$ a.e. on $(0, T) \times \partial \Omega$ :

$$
\begin{gathered}
\int_{0}^{T} \int_{\Omega}\left\{\nabla\left(\int_{s}^{X} F(\eta) d \eta\right)^{+} \cdot \nabla \psi-(\mathbf{G}(\max \{X, s\})-\mathbf{G}(s)) \cdot \nabla \psi-\right. \\
\left.\phi(X-s)^{+} \partial_{t} \psi\right\} d x d t-\left.\int_{\Omega} \phi\left(X_{0}-s\right)^{+} \psi\right|_{t=0}-M \int_{0}^{T} \int_{\partial \Omega}\left(X_{D}-s\right)^{+} \psi d \gamma(x) d t \leq 0, \\
\int_{0}^{T} \int_{\Omega}\left\{\nabla\left(\int_{s}^{X} F(\eta) d \eta\right)^{-} \cdot \nabla \psi+(\mathbf{G}(\min \{X, s\})-\mathbf{G}(s)) \cdot \nabla \psi-\right. \\
\left.\phi(X-s)^{-} \partial_{t} \psi\right\} d x d t-\left.\int_{\Omega} \phi\left(X_{0}-s\right)^{-} \psi\right|_{t=0}-M \int_{0}^{T} \int_{\partial \Omega}\left(X_{D}-s\right)^{-} \psi d \gamma(x) d t \leq 0
\end{gathered}
$$

After [29], page 2267, Corollary 4.1, we know that under hypothesis (H1)(H6 ) there exists a unique weak entropy solution. For more results we refer to [4], [3], [5] and [28].

In this article we are concerned with calculating the weak entropy solution. A widely used approach is to perform the change of the unknown function, using the Kirchhoff transform. It requires the strict positivity of $F$, which is equivalent to the invertibility of $\int_{0}^{X} F(\eta) d \eta$. In our case, $\int_{0}^{X} F(\eta) d \eta$ is not continuously invertible. The practical remedy to this difficulty is to 
regularize $F$, in order to have it strictly positive. For such regularized problem, we know after [13], page 331, that every weak solution is an entropy weak solution and, consequently, unique.

For the case without capillary pressure, we will use two different regularization approaches to determine the entropy solution. The first approach consists in letting the parameter $P_{r}$ in the van Genuchten expression (or $P_{c}^{M}$ for the linear case) go to 0 . The second one is achieved by regularizing the expression of $F(X)$ without introducing $\widehat{P}_{c}$. In order to have a non-negative $F(X)$ defined everywhere, we extend it by continuity for the values $X \leqslant 0$. The regularization is given by

$$
F_{\tau}(X)= \begin{cases}\frac{\mathfrak{r} T K}{\mu_{g}} \tau, & \text { if } X<0, \\ \frac{\mathfrak{r} T K}{\mu_{g}}(X+\tau), & \text { if } 0 \leqslant X \leqslant \rho_{g}^{*}, \\ \frac{\mathfrak{r} T K}{\mu_{g}}\left(\rho_{g}^{*}+\tau\right)+\frac{\mathfrak{r} T K \rho_{g}^{*}}{\mu_{g}} \frac{\rho_{g}^{*}-X}{\tau} & \text { if } \rho_{g}^{*}<X \leqslant \rho_{g}^{*}+\tau, \\ \frac{\mathfrak{r} T K}{\mu_{g}} \tau, & \text { if } \rho_{g}^{*}+\tau<X \leqslant \rho_{l}^{*}-\tau, \\ \left(\frac{\mathfrak{r} T K}{\mu_{g}}-\frac{K}{\alpha \mu_{l} \tau}\right)\left(\rho_{l}^{*}-X\right)+\frac{K}{\alpha \mu_{l}}, & \text { if } \rho_{l}^{*}-\tau<X<\rho_{l}^{*}, \\ \frac{K}{\alpha \mu_{l}}, & \text { if } \quad X \geqslant \rho_{l}^{*},\end{cases}
$$

with $\tau>0$. This procedure can be justified using the results from [12], Theorem 1.1. The stiffness of the problem solved in the paper with respect to the small parameter $\tau$ is discussed in Subsection 3.4. A direct approach to solve the non regularized problem using a monotone Finite Volume scheme is in the articles [19] and [29]. Concerning the situation with non-negligible capillary pressure, there are many references when the diffusive $F$ vanishes after a threshold valued. We mention the reference $[6,17,18,22,33,36,37,38,39]$. In our approach we allow both degenerate parabolic and hyperbolic situations.

In Figure 2 we depicted the qualitative behavior of $F(X)$ and the capillary pressure curves in various cases.

Finally, we regularize in both cases (with and without capillarity) $F$ in a neighborhood of $X=0$.

The equation (20) is further transformed using the Kirchhoff transformation, i.e. by introducing a nonlinear transformation (see also Figure 2)

$$
Y:[0,+\infty) \rightarrow \mathbb{R}, \quad X \rightarrow Y(X)=\int_{0}^{X} F(z) d z .
$$




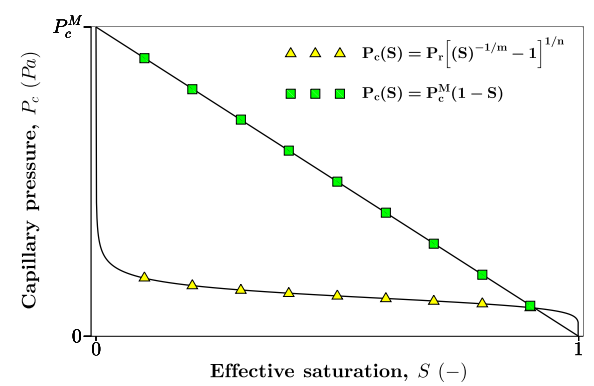

Capillary Pressures

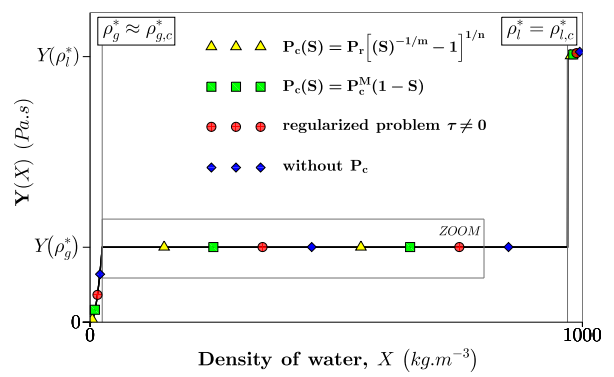

Graphs of $Y(X)$

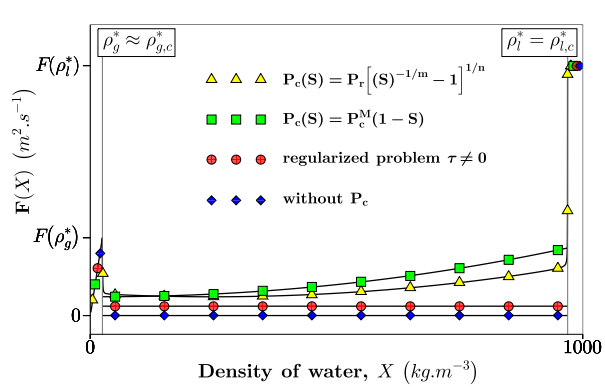

Graphs of $F(X)$

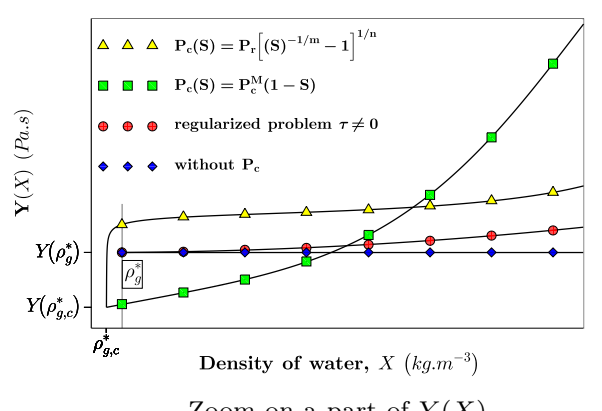

Zoom on a part of $Y(X)$

Figure 2: Qualitative behaviors of the capillarity pressure functions, and the graphs of $F(X)$ and of $Y(X)$.

Notice that, after considering $F$ given by (48) (respectively by (41)) for $\rho_{g}^{*}<$ $X<\rho_{l}^{*}$ (respectively $\rho_{g, c}^{*}<X<\rho_{l, c}^{*}$ ), we find out that $Y(X)$ is invertible for $X \in \mathbb{R}$. Equation (20) becomes

$$
\phi \frac{\partial X(Y)}{\partial t}-\Delta Y+\nabla \cdot \mathbf{G}(X(Y))=0 .
$$

In both cases (with and without capillary effects), we have introduced a global equation, formulated through a single variable $X$ which physically represents the $\mathrm{H}_{2} \mathrm{O}$ density within the medium. In the zone $\rho_{g}^{*}<X<\rho_{l}^{*}$ (or $\rho_{g, c}^{*}<X<\rho_{l, c}^{*}$ ) liquid and vapor are simultaneously present in the porous medium.

\section{Numerical simulations}

In this section we present the simulations for four test cases.

The first test case is performed in two independent ways, one using the software COMSOL Multiphysics (in 1D) and the other using the numerical model given in (52) and implemented under the MmodD-Scilab project [31]. For all other tests the second approach was used. The first and second test cases are 
quite similar, in the first we have considered no flux boundary conditions and in the second Dirichlet boundary conditions. The third test case aims to illustrate the influence of the isothermal mechanical compressibility factor $\alpha$. Finally, the last test case consists in applying our scheme to a more realistic situation.

\subsection{Discrete scheme used in the MmodD-Scilab implementation}

Let $\Omega$ be a bounded domain in $\mathbb{R}^{2}$ with a Lipschitz continuous boundary $\partial \Omega$. Let $\Gamma_{D}$ and $\Gamma_{N}$ be disjoint subsets of the boundary so that $\partial \Omega=\Gamma_{D} \cup \Gamma_{N}$.

We are interested in solving (50) with following initial and boundary conditions

$$
\begin{aligned}
& Y=Y_{D} \quad \text { on } \quad \Gamma_{D} \times(0, T), \\
& \begin{aligned}
(\nabla Y+\mathbf{G}(X(Y))) \cdot \nu & =g & & \text { on } \quad \Gamma_{N} \times(0, T), \\
Y_{\left.\right|_{t=0}} & =Y_{0}(x) & & \text { on } \Omega,
\end{aligned}
\end{aligned}
$$

where $(0, T)$ is the time interval, $T>0$, and $\nu$ is the outer normal of $\partial \Omega$.

The numerical approach employs $P 1$ finite elements in space and the Euler semi-explicit scheme in time. We use mass lumping approach [33]-[34] with weakly-acute mesh to garanty the monotonicity. For the diffusion part we use the implicit scheme and for the non-linear term in the time derivative the explicit scheme. With $N>0$ integer, set $\delta t=T / N$ and let $\mathcal{T}_{h}$ be a decomposition of $\Omega$ into a triangular mesh. $h$ stands for the mesh size. Now the fully discrete $P 1$ finite element approximation of problem (50), (51) reads as follows:

$Y_{h}^{0}$ is given by $Y_{h}^{0}:=\Pi_{h} Y_{0}$,

for $n=0, . ., N-1$, find $Y_{h}^{n+1}-\Pi_{h} Y_{D} \in V_{h}$ such that for all $v_{h} \in V_{h}$ :

$$
\begin{aligned}
& \int_{\Omega} \Pi_{h}\left(\frac{\phi}{F\left(X\left(Y_{h}^{n}\right)\right)} \frac{Y_{h}^{n+1}-Y_{h}^{n}}{\delta t} v_{h}\right) d x+\int_{\Omega} \nabla Y_{h}^{n+1} \nabla v_{h} d x \\
& +\int_{\Omega} G\left(X\left(Y_{h}^{n+1}\right)\right) \nabla v_{h} d x-\int_{\Gamma_{N}} g v_{h} d \Gamma=0,
\end{aligned}
$$

where $\Pi_{h}$ is the Lagrange interpolation operator and $V_{h}$ the $P 1$ linear continuous finite element space.

Concerning the time stepping, we consider an automatic time-step control algorithm. Many papers describe the method for constructing adaptive timestep, see for example [25]. We will use the error per unit step method, for which we seek to control for a given error tolerance $\varepsilon_{T}$

$$
\left\|Y_{h}^{n+1}\left(\delta t=\delta t_{n}\right)-Y_{h}^{n+1}\left(\delta t=0.5 \delta t_{n}\right)\right\|_{L^{2}\left(t_{n}, t_{n+1} ; L^{2}(\Omega)\right)} \leqslant \varepsilon_{T},
$$

where $Y_{h}^{n+1}\left(\delta t=\delta t_{n}\right)$ and $Y_{h}^{n+1}\left(\delta t=0.5 \delta t_{n}\right)$ are the solutions of $(52)$ for $\delta t=$ $\delta t_{n}$ and $\delta t=0.5 \delta t_{n}$, respectively. More precisely, knowing $\delta t_{n}$ and two strictly positive small parameters $\varepsilon_{\text {sup }}$ and $\varepsilon_{\text {inf }}$, we calculate $Y_{h}^{n+1}$ for $\delta t=\delta t_{n}$ and $\delta t=0.5 \delta t_{n}$ and the error

$$
\varepsilon_{n}=\frac{\left\|Y_{h}^{n+1}\left(\delta t=\delta t_{n}\right)-Y_{h}^{n+1}\left(\delta t=0.5 \delta t_{n}\right)\right\|_{L^{2}\left(t_{n}, t_{n+1} ; L^{2}(\Omega)\right)}}{\left\|Y_{h}^{n+1}\left(\delta t=0.5 \delta t_{n}\right)\right\|_{L^{2}\left(t_{n}, t_{n+1} ; L^{2}(\Omega)\right)}} .
$$


Then

- if $\varepsilon_{n}>\varepsilon_{\text {sup }}$, we recalculate $Y_{h}^{n+1}$ with $\delta t_{n}=(2 / 3) \delta t_{n}\left(\varepsilon_{\text {sup }} / \varepsilon_{n}\right)$,

- if $\varepsilon_{\text {inf }}<\varepsilon_{n} \leqslant \varepsilon_{\text {sup }}$, we proceed to the next step with $\delta t_{n+1}=\delta t_{n}$,

- else $\left(\varepsilon_{n}<\varepsilon_{i n f}\right)$, we proceed to the next step with $\delta t_{n+1}=\delta t_{n}\left(\varepsilon_{i n f} / \varepsilon_{n}\right)$.

\subsection{COMSOL Multiphysics}

Other numerical method we used for (20) is based on the finite element method (FEM) and the backward Euler method for the time stepping. To implement these techniques we used the software COMSOL Multiphysics [15]. COMSOL Multiphysics is a software package for solving and analyzing physical problems through the extensive and well organized interface of Matlab. The input data are at Table 3, while Table 2 briefly summarizes the COMSOL modules used for the simulation. The results, which concern the Test case 1, are presented in Figure 3.

\begin{tabular}{|ll|}
\hline Numerical details & \\
\hline Finite element & Lagrangian quadratic \\
Degrees of freedom & 961 \\
Linear solver & PARDISO (direct) \\
\hline
\end{tabular}

Table 2: Numerical choices.

3.3. Test case 1 : no flux boundary conditions

\begin{tabular}{|l|l||l|l|}
\hline \multicolumn{2}{|c||}{ Fluid and medium parameters } & \multicolumn{2}{c|}{ Other parameters } \\
\hline Parameter & Value & Parameter & Value \\
\hline$P_{l}^{o}$ & $7 \mathrm{MPa}$ & $L$ & $2 \mathrm{~km}$ \\
$\rho_{l}^{O}$ & $900 \mathrm{~kg} \cdot \mathrm{m}^{-3}$ & $g$ & $9.81 \mathrm{~m} \cdot \mathrm{s}^{-2}$ \\
$\mu_{l}$ & $10^{-3} \mathrm{~Pa} . \mathrm{s}$ & $k_{r, l}(\mathrm{~S})$ & $\mathrm{S}^{3}$ \\
$\mu_{g}$ & $1,5.10^{-5} \mathrm{~Pa} . \mathrm{s}$ & $k_{r, g}(1-S)$ & $1-S^{3}$ \\
$\alpha$ & $7,5.10^{-10} \mathrm{~Pa}^{-1}$ & $\hat{P}_{c}(S)$ & $P_{c}^{M}(1-S)$ \\
$T$ & $553 \mathrm{~K}$ & \\
$\mathfrak{r}$ & $461.89{\mathrm{~J} . \mathrm{kg}^{-1} . \mathrm{K}^{-1}}$ & & \\
$K$ & $10^{-13} \mathrm{~m}^{2}$ & & \\
$\phi$ & 0.4 & & \\
\hline
\end{tabular}

Table 3: Numerical parameters.

In addition to Comsol Multiphysics simulation, the $2 \mathrm{D}$ simulation using MmodD [31] is performed. The spatial domain is the interval $[-L, 0] \times[0, H]$, with $L=2000 \mathrm{~m}$. We suppose initial data depending only on the first variable and the no-flux condition on lateral boundary. Consequently, the solution depends only on the first variable and the problem is in fact 1D. This allow comparison with the 1D case. The numerical parameters for this simulation are given in the Table 3. Initially, the lower part of the domain was saturated 


\begin{tabular}{|c|c|}
\hline$\overline{P_{c}^{M}} \quad(P a)$ & $\begin{array}{ll}\rho_{\text {c }}^{*} & \left(\mathrm{~kg} \cdot \mathrm{m}^{-3}\right)\end{array}$ \\
\hline $10^{5}$ & $\frac{g, c}{25.44}$ \\
\hline $10^{6}$ & 25.34 \\
\hline $10^{7}$ & 24.37 \\
\hline $10^{8}$ & 16.48 \\
\hline
\end{tabular}

Table 4: Phase transition values with respect to $P_{c}^{M}$.
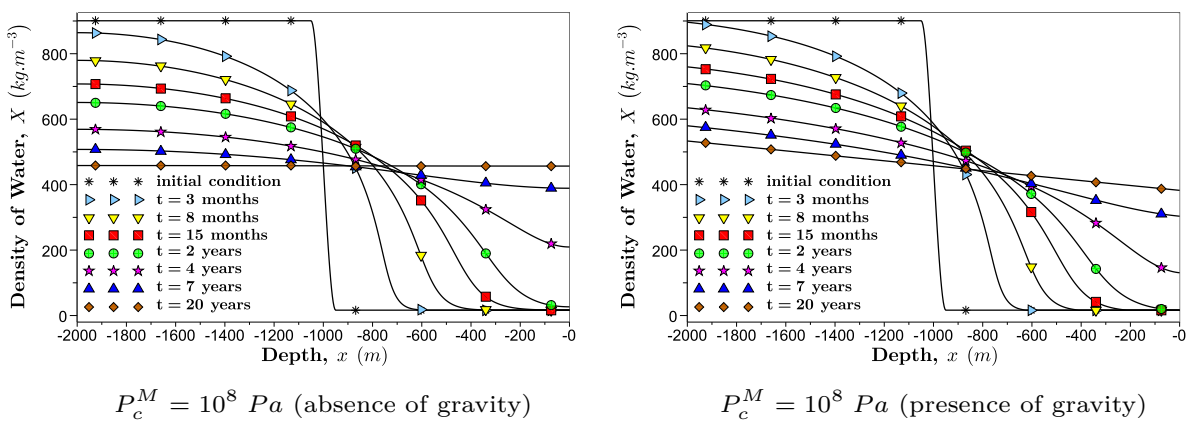

Figure 3: $P_{c}^{M}=P a, 10^{8} P a$. Spatial evolution of the density of water $(X)$ at several times. The left column corresponds to the case without gravity and the right column corresponds to the case with gravity.

by liquid $\mathrm{H}_{2} \mathrm{O}$, while the upper part contained only vapor. Consequently the initial condition for $X$ is the Heaviside function centered in $x=-L / 2$. The function is regularized by a cubic spline. This means that there is a zone at the middle of the domain in which the fluid is a mixture of vapor and liquid. The boundary conditions are the no-flux conditions and as initial conditions, we consider different profiles of the density of water for the different values of $P_{c}^{M}$

$$
X_{0}(x)= \begin{cases}\rho_{l, c}^{*}+1\left(\rho_{l, c}^{*}=\rho_{l}^{*}\right), & \text { if } \quad x \in[-L,-1050 \mathrm{~m}], \\ \text { regularization (cubic spline), } & \text { if } \quad x \in[-1050 \mathrm{~m},-950 \mathrm{~m}], \\ \rho_{g, c}^{*}-\frac{\rho_{g, c}^{*}}{\rho_{l}^{*}}, & \text { if } \quad x \in[-950 \mathrm{~m}, 0] .\end{cases}
$$

Since $\widehat{P}_{c}(1)=0$, we have $\rho_{l, c}^{*}=\rho_{l}^{*} \approx 899.66 \mathrm{~kg} \cdot \mathrm{m}^{-3}$. The values of $\rho_{g, c}^{*}$, corresponding to different values of $P_{c}^{M}$, are given in the Table 4 .

The results of the simulation using COMSOL and MmodD are impossible to distinguish and they are depicted in Fig. 3-4. On the left part of this figure we can see the simulation corresponding to the case with no gravity. We may remark that reducing $P_{c}^{M}$ (see Figure 4), the gravitational force causes the clear separation between the liquid and gaseous phases. 

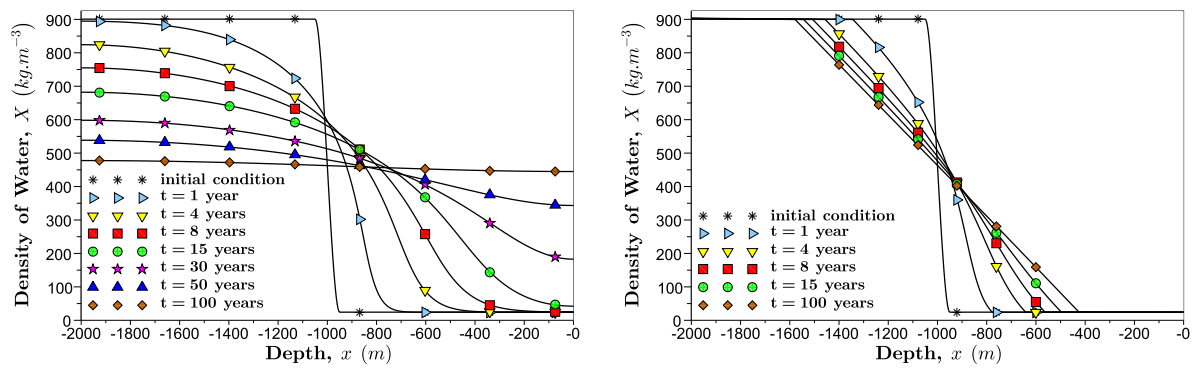

$P_{c}^{M}=10^{7} \mathrm{~Pa}$ (absence of gravity)

$P_{c}^{M}=10^{7} \mathrm{~Pa}$ (presence of gravity)
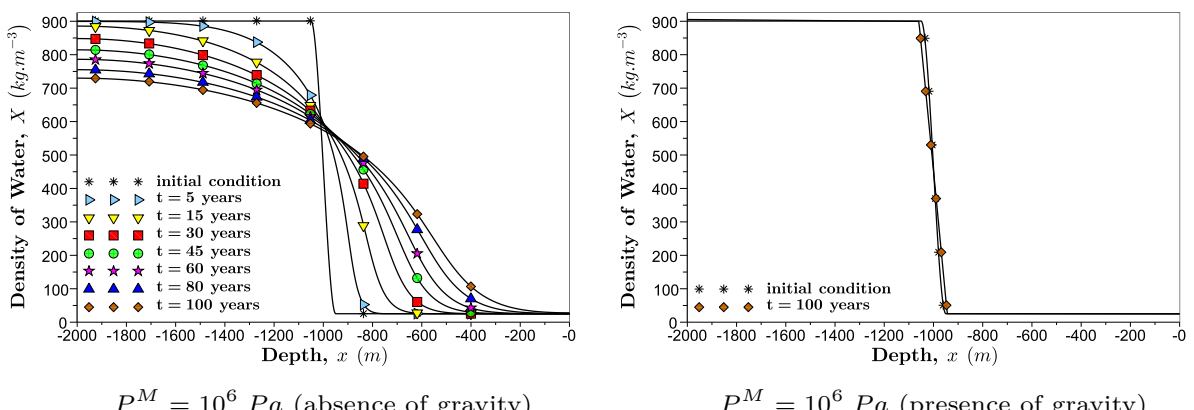

$P_{c}^{M}=10^{6} P a$ (presence of gravity)
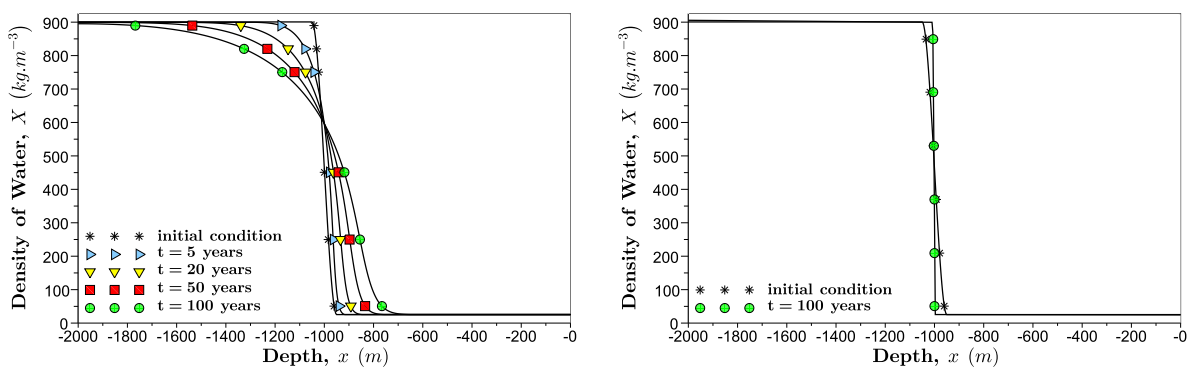

$P_{c}^{M}=10^{5} P a$ (absence of gravity)

$P_{c}^{M}=10^{5} P a$ (presence of gravity)

Figure 4: $P_{c}^{M}=\left[10^{5} \mathrm{~Pa}, 10^{6} \mathrm{~Pa}, 10^{7} \mathrm{~Pa}\right]$. Spatial evolution of the density of water $(\mathrm{X})$ at several times. The left column corresponds to the case without gravity and the right column corresponds to the case with gravity.

\subsection{Test case 2: Dirichlet boundary conditions}

The spatial domain is $\Omega=[0,1000] \times[0,100]$ meters, with a mesh size $h=5 / 3 \mathrm{~m}$. The initial configuration is the same as in the previous test case, uniformly with respect to $y$. We neglect the gravitational effects. The numerical parameters are given in the Table 5 . For the time discretization, we choose an initial time-step of $\delta t_{0}=0.1 \mathrm{~s}$, and take $\varepsilon_{\text {sup }}=2 \times 10^{-5}, \varepsilon_{\text {inf }}=10^{-5}$.

The aim of this test case is to show the influence of the capillary effects in 


\begin{tabular}{|l|l||l|l|}
\hline \multicolumn{2}{|c||}{ Fluid and medium parameters } & \multicolumn{2}{c|}{ VG-Mualem parameters } \\
\hline Parameter & Value & Parameter & Value \\
\hline$P_{l}^{o}$ & $7 \mathrm{MPa}$ & $n$ & $6.6($ see $[41])$ \\
$\rho_{l}^{o}$ & $970 \mathrm{~kg} . \mathrm{m}^{-3}$ & $m$ & $1-1 / n$ \\
$\mu_{l}$ & $10^{-3} \mathrm{Pa.s}$ & $k_{r, l}(S)$ & $\sqrt{S}\left[1-\left(1-S^{1 / m}\right)^{m}\right]^{2}$ \\
$\mu_{g}$ & $1,5.10^{-5} \mathrm{Pa.s}$ & $k_{r, g}(1-S)$ & $1-k_{r, l}(S)$ \\
$\alpha$ & $7,5.10^{-10} \mathrm{~Pa}^{-1}$ & $\widehat{P}_{c}(S)$ & $P_{r}\left[(S)^{-1 / m}-1\right]^{1 / n}$ \\
$T$ & $553 \mathrm{~K}^{-1}$ & & \\
$\mathfrak{r}$ & $461.89 \mathrm{Jgg}^{-1} \cdot \mathrm{K}^{-1}$ & & \\
$K$ & $10^{-12} \mathrm{~m}^{2}$ & & \\
$\phi$ & 0.4 & & \\
\hline
\end{tabular}

Table 5: Numerical parameters.

\begin{tabular}{|c|c|cc|}
\hline$P_{r} \quad(\mathrm{~Pa})$ & $P_{c}^{M} \quad(\mathrm{~Pa})$ & $\rho_{g, c}^{*}$ & $\left(\mathrm{~kg} . \mathrm{m}^{-3}\right)$ \\
\hline $1.26 \times 10^{4}$ & $10^{5}$ & 25.44 \\
$1.26 \times 10^{5}$ & $10^{6}$ & \multicolumn{2}{c|}{25.38} \\
$6.3 \times 10^{5}$ & $5 \times 10^{6}$ & \multicolumn{2}{|c|}{54.94} \\
\hline
\end{tabular}

Table 6: Phase transition values with respect to $P_{r}$ and $P_{c}^{M}$.

\begin{tabular}{|l|l|c|c|}
\hline Conditions & Domain, boundary & regularized model & model with capillarity \\
\hline \multirow{3}{*}{ Initial } & $x \in[0,450]$ & $\rho_{l}^{*}+2 / 5$ & $\rho_{l, c}^{*}+2 / 5$ \\
& $x \in[450,550]$ & \multicolumn{2}{|c|}{ regularization by a cubic spline } \\
& $x \in[550,1000]$ & $\rho_{g}^{*}-2 / 5$ & $\rho_{g, c}^{*}-2 / 5$ \\
\hline \multirow{5}{*}{ Boundary } & $\left.\Gamma_{t}=\right] 0,1000[\times\{100\}$ & no flux condition & no flux condition \\
& $\left.\Gamma_{b}=\right] 0,1000[\times\{0\}$ & no flux condition & no flux condition \\
& $\Gamma_{\text {in }}=\{0\} \times[0,100]$ & $X=\rho_{l}^{*}+2 / 5$ & $X=\rho_{l, c}^{*}+2 / 5$ \\
& $\Gamma_{\text {out }}=\{1000\} \times[0,100]$ & $X=\rho_{g}^{*}-2 / 5$ & $X=\rho_{g, c}^{*}-2 / 5$ \\
\hline
\end{tabular}

Table 7: Initial and boundary conditions. Due to the symmetry, $X_{0}(x, y)=X_{0}(x)$ for all $y \in[0,100]$.

comparison with the regularized model (48). We will vary the values of the parameter $P_{r}$ in the regularized expression of van Genuchten and the values of $\tau$. Since $\widehat{P}_{c}(1)=0$, we have $\rho_{l, c}^{*}=\rho_{l}^{*} \approx 899.66 \mathrm{~kg} \cdot \mathrm{m}^{-3}$. For the phase transition gas/liquid + gas, we have $\rho_{g}^{*}=25.45 \mathrm{~kg} \cdot \mathrm{m}^{-3}$, and the values of $\rho_{g, c}^{*}$, corresponding to different values of $P_{r}$ in the capillary pressure function with $P_{c}^{M}=(10 / 1.26) P_{r}$, are given in the Table 6.

As initial condition we consider different profiles of the density of water, and we impose Dirichlet and Neumann boundary conditions, see Table 7.

Saturation $S$ and water density $X$ at various time-steps, for different values of the regularization parameter $\tau$ and different values of $P_{r}$, are shown in Figures 5 and 6 , respectively. Note that the different solutions converge to a stationary solution. As can be seen in the figures, the saturation and density of water have similar profiles from the qualitative point of view. On the other hand, when the capillary effects in the unsaturated zone become negligible, then we 

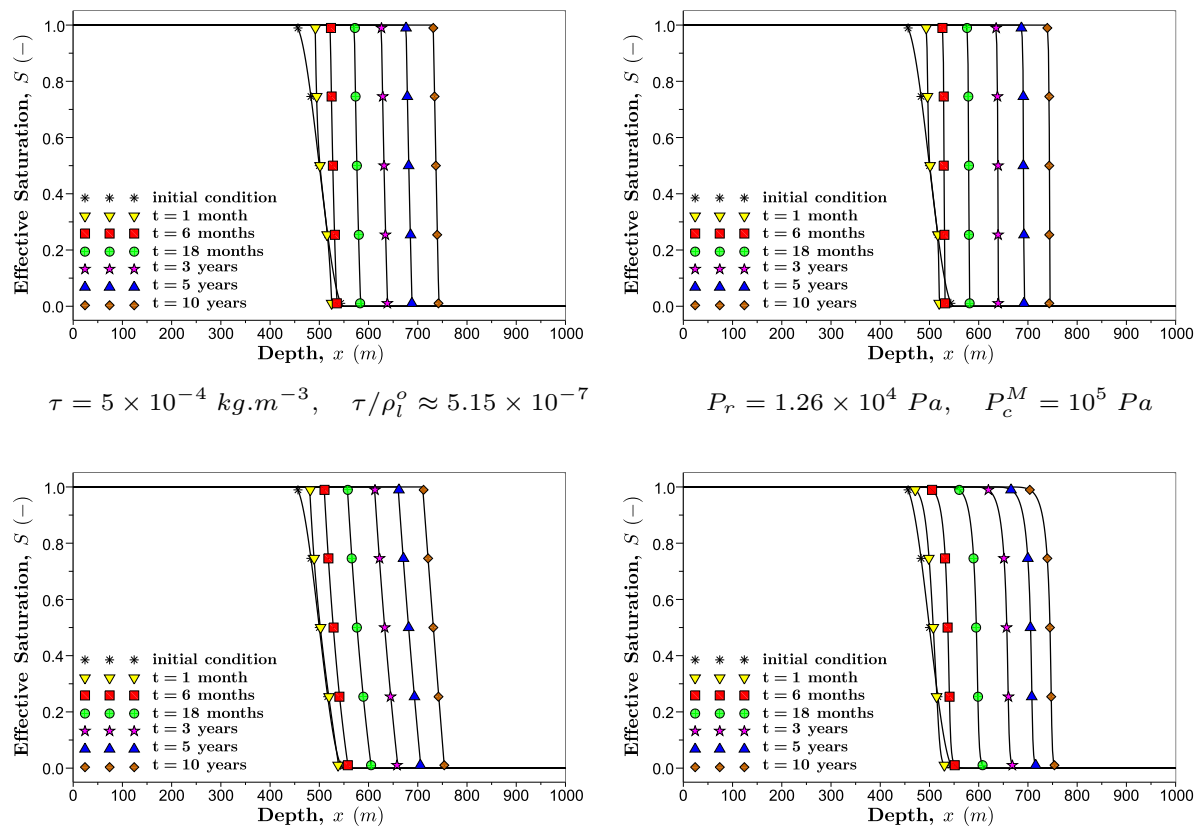

$P_{r}=1.26 \times 10^{4} \mathrm{~Pa}, \quad P_{c}^{M}=10^{5} \mathrm{~Pa}$

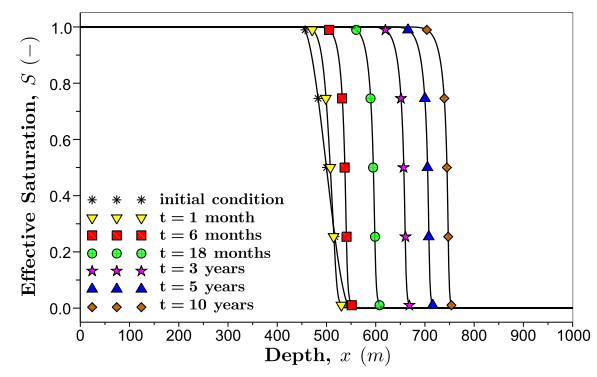

$\tau=2 \times 10^{-3} \mathrm{~kg} \cdot \mathrm{m}^{-3}, \quad \tau / \rho_{l}^{o} \approx 2.06 \times 10^{-6}$

$$
P_{r}=1.26 \times 10^{5} \mathrm{~Pa}, \quad P_{c}^{M}=10^{6} \mathrm{~Pa}
$$
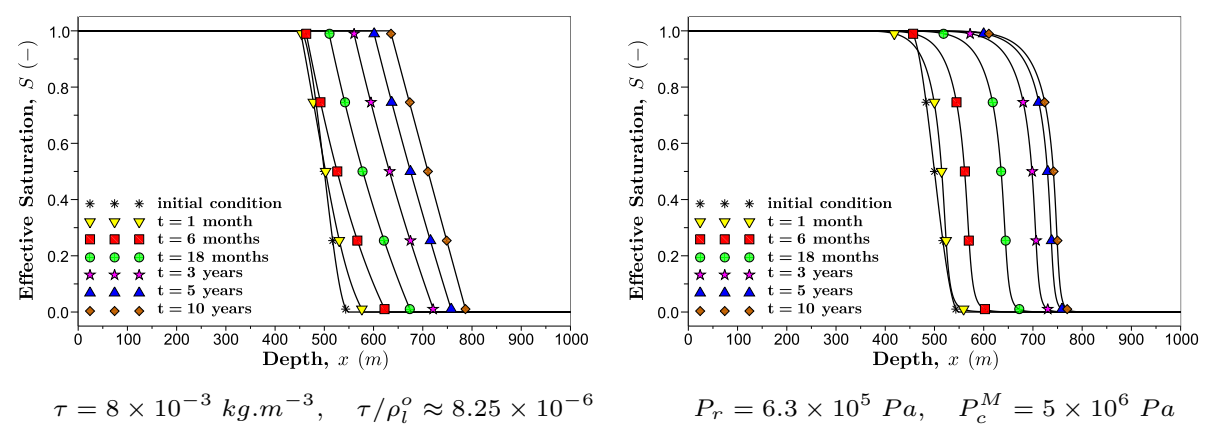

Figure 5: Effective saturation spatial evolution at $y=50 \mathrm{~m}$ for several times. The left three figures correspond to the regularized problem with $\tau=$ $\left[5 \times 10^{-4}, 2 \times 10^{-3}, 8 \times 10^{-3}\right] \quad \mathrm{kg}^{-\mathrm{m}^{-3}}$. Whereas the right three figures correspond to the model with capillarity for the capillary function given in Table 5 with $P_{r}=$ $\left[1.26 \times 10^{4}, 1.26 \times 10^{5}, 6.3 \times 10^{5}\right] P a$.

observe the front propagation and a clear separation between the liquid and gaseous phases. We note that the well-posedness frame for this kind of problem (hyperbolic equations) is in [5] and [11].

The capillary effects can be finally interpreted as a regularization of the model where capillary effect are neglected. To complete the discussion one 

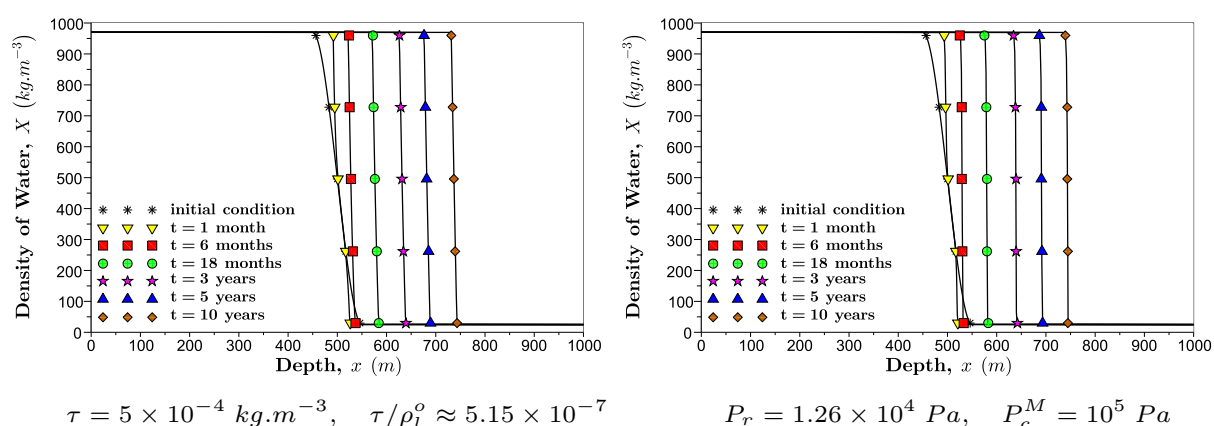

$\tau=5 \times 10^{-4} \mathrm{~kg} \cdot \mathrm{m}^{-3}, \quad \tau / \rho_{l}^{o} \approx 5.15 \times 10^{-7}$

$P_{r}=1.26 \times 10^{4} \mathrm{~Pa}, \quad P_{c}^{M}=10^{5} \mathrm{~Pa}$
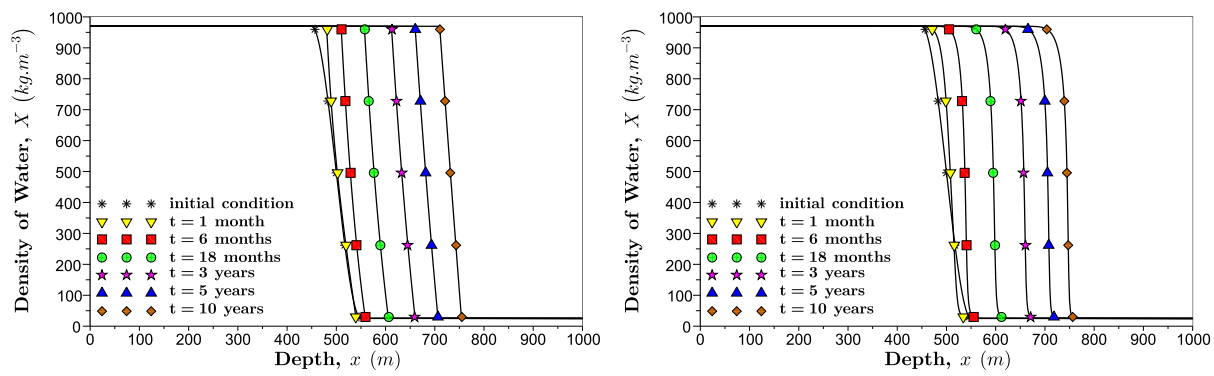

$\tau=2 \times 10^{-3} \mathrm{~kg} \cdot \mathrm{m}^{-3}, \quad \tau / \rho_{l}^{o} \approx 2.06 \times 10^{-6}$

$P_{r}=1.26 \times 10^{5} \mathrm{~Pa}, \quad P_{c}^{M}=10^{6} \mathrm{~Pa}$
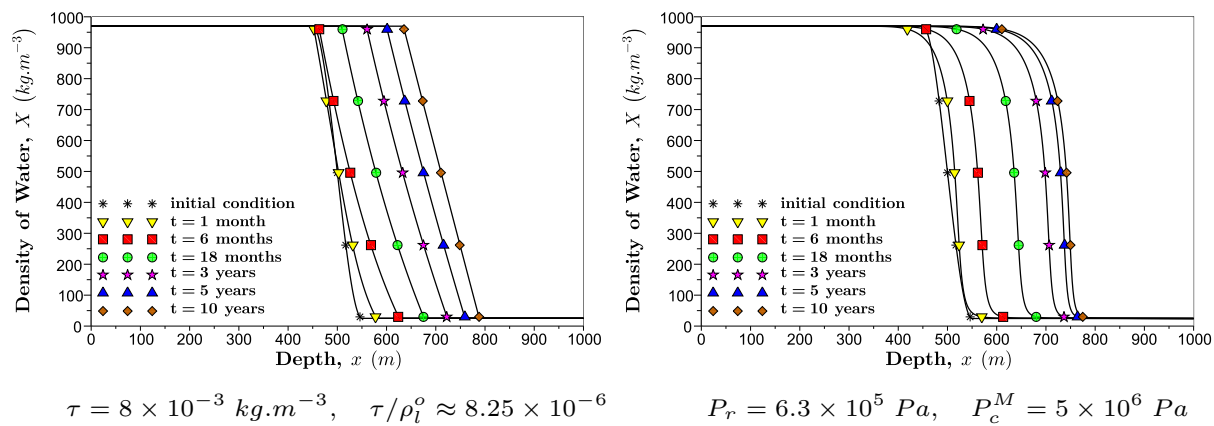

Figure 6: Water density spatial evolution at $y=50 \mathrm{~m}$ for several times. The left three figures correspond to the regularized problem with $\tau=\left[5 \times 10^{-4}, 2 \times 10^{-3}, 8 \times 10^{-3}\right] \mathrm{kg} \cdot \mathrm{m}^{-3}$. Whereas the right three figures correspond to the model with capillarity for the capillary function given in Table 5 with $P_{r}=\left[1.26 \times 10^{4}, 1.26 \times 10^{5}, 6.3 \times 10^{5}\right] P a$.

would like to check numerically that the model with diffusion $\left(\tau>0\right.$ or $\left.\widehat{P}_{c} \neq 0\right)$ converges to the solution of the model whith no capillary effects. The Figure 7 illustrate this situation. Indeed, we represent on this figure the difference between the approximate solutions of two consecutive elements of a sequence that converges to 0 , where the elements are: the regularization parameters $\tau$ or $P_{c}^{M}$. This figure is obtain with fixed dimensionless discretization parameters, 
$\widetilde{\delta}_{t}=\delta t / T \approx 3.1 \times 10^{-5}$ and $\widetilde{h}=h / L \approx 2 \times 10^{-3}$ for a simulation time of 3 days and a domain length of $1 \mathrm{~km}$. As expected, one observes a straight line in logarithmic scale when $\tau$ or $P_{c}^{M}$ are sufficiently small. In the absence of the benchmark, the observed results confirmed validity of the method.

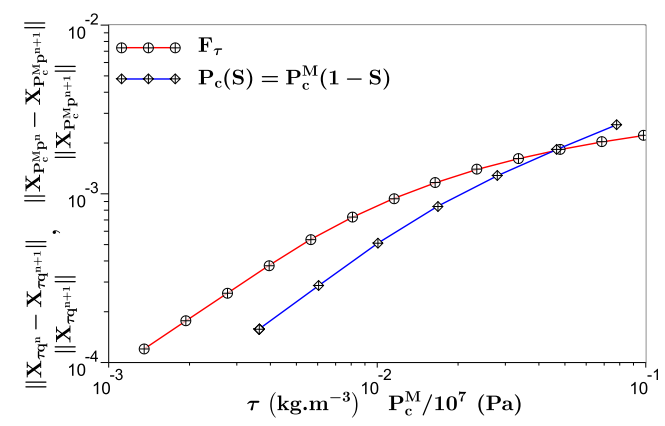

Figure 7: Normalized $L^{2}\left(0, T, L^{2}(\Omega)\right)$ difference between consecutive solutions in log-scale

\subsection{Influence of the isothermal mechanical compressibility factor}

We consider now a smaller domain, given by $\Omega=[0,100] \times[0,10] m$, with a mesh size $h=1 / 8 \mathrm{~m}$. This test case is intended to show the small effect of the mechanical compressibility factor $\alpha$ on the flow model, considering different values of it. We will take an initial condition close to 1 for the saturation and Neumann boundary conditions.

Concerning the time-step control algorithm, we consider an initial time-step of $\delta t_{0}=10^{-2} \mathrm{~s}$, and take $\varepsilon_{\text {sup }}=10^{-6}, \varepsilon_{\text {inf }}=10^{-7}$. As choice for initial condition, we set $X=950 \mathrm{~kg} \mathrm{~m}^{-3}$, which corresponds to $S$ between 0.98306 and 0.98405 for the different values of $\alpha$. We use same material parameters as in previous section, i.e., $\phi=0.4$ and $K=10^{-12} \mathrm{~m}^{2}$. Furthermore we consider the presence of capillarity effects (see Table 5 with $P_{r}=1.26 \times 10^{6} \mathrm{~Pa}$ ). We impose the boundary conditions from Table 7 on $\Gamma_{t}$ and $\Gamma_{b}$. On $\Gamma_{i n}$, we impose $X=950$, and on $\Gamma_{\text {out }}$, we impose

$$
F(X) \nabla X \cdot \nu=-0.14 \text { Pa.s. } m^{-1} .
$$

Water density at the time $t=1$ hour is depicted in Figure 8 for $\alpha \in$ $\left[10^{-9}, 5.10^{-10}, 10^{-10}, 10^{-16}\right]$. As physically expected, $\alpha$ has a negligible influence on the result of the simulation. Based on theses results and the values of $\alpha$ found in the literature : $\alpha=7,5.10^{-10} \mathrm{~Pa}^{-1}$ (see [41]), $\alpha=4.9 .10^{-10} \mathrm{~Pa}^{-1}$ (see $[26]$ ); one can consider that taking into account the mechanical compressibility of the water in the model has little influence on the dynamics. 

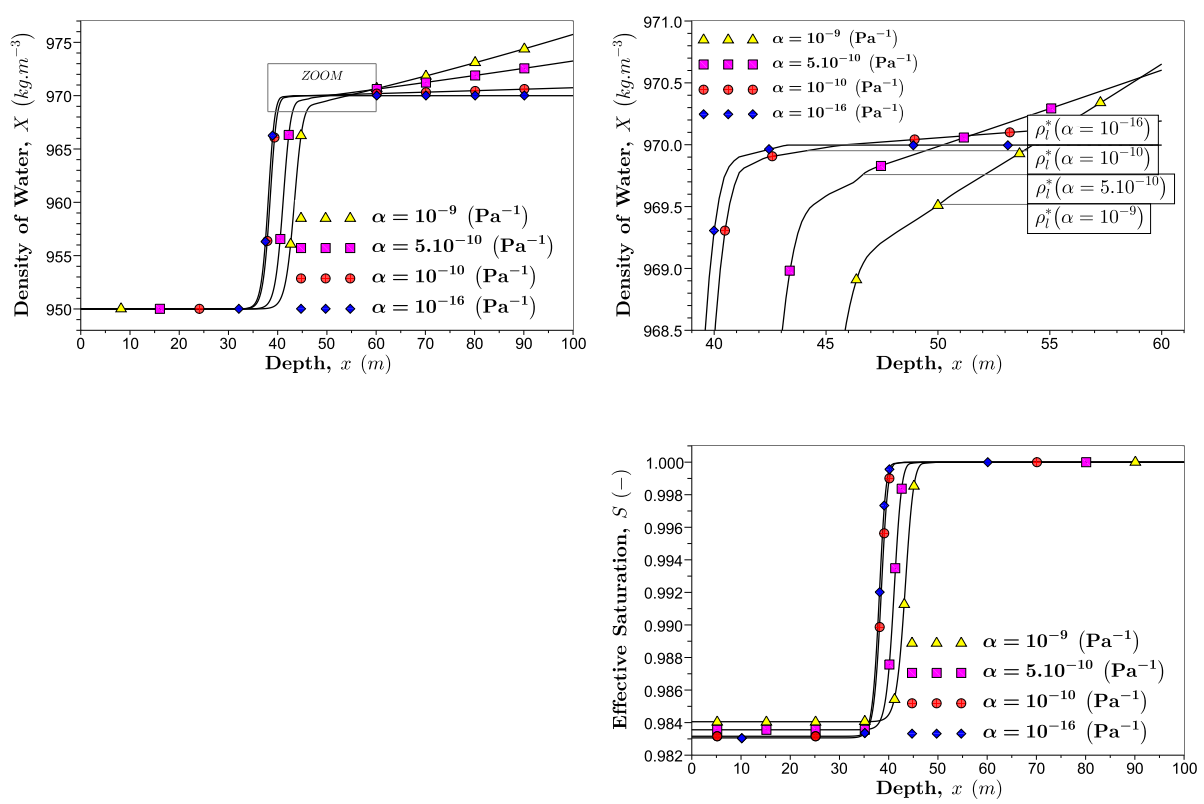

Figure 8: Spatial evolution along the line $[0,100] \times\{5\}$ of $X$ and $S$ at the time $t=1$ hour for $\alpha \in\left[10^{-9}, 5.10^{-10}, 10^{-10}, 10^{-16}\right] \mathrm{Pa}^{-1}$; where $X$ is the density of water and $S$ the effective liquid saturation.
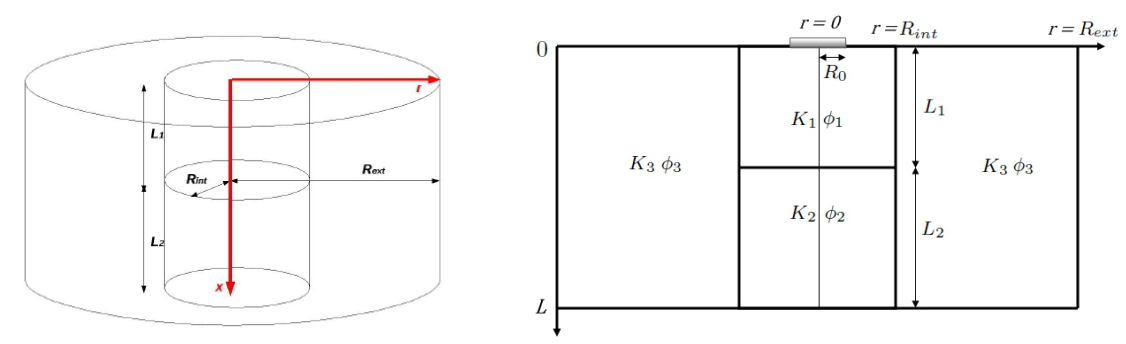

Figure 9: Domain geometry.

\begin{tabular}{|l|l|l|l||l|l|l|l|}
\hline \multicolumn{4}{|c||}{ Geometry parameters } & \multicolumn{4}{c|}{ Medium parameters } \\
\hline Quantity & Value & Quantity & Value & Quantity & Value & Quantity & Value \\
\hline$L_{1}$ & $1 \mathrm{~km}$ & $R_{\text {int }}$ & $2 \mathrm{~km}$ & $K_{1}$ & $10^{-13} \mathrm{~m}^{2}$ & $\phi_{1}$ & 0,4 \\
$L_{2}$ & $1 \mathrm{~km}$ & $R_{\text {ext }}$ & $8 \mathrm{~km}$ & $K_{2}$ & $10^{-14} \mathrm{~m}^{2}$ & $\phi_{2}$ & 0,2 \\
& & $R_{0}$ & $200 \mathrm{~m}$ & $K_{3}$ & $10^{-18} \mathrm{~m}^{2}$ & $\phi_{3}$ & 0,1 \\
\hline
\end{tabular}

Table 8: Geometry and medium parameters 

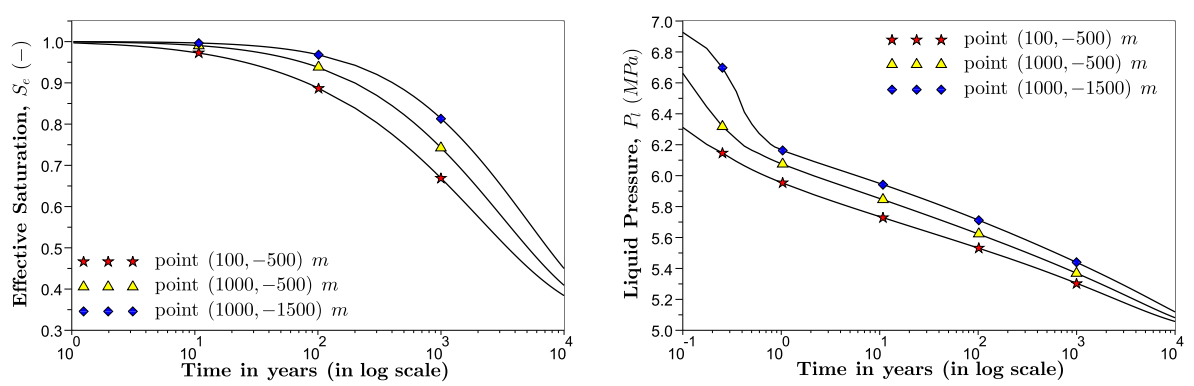

Figure 10: Time evolution at the points $(100,-500) m,(1000,-500) m$, and $(1000,-1500) m$ of the effective saturation and the liquid pressure.

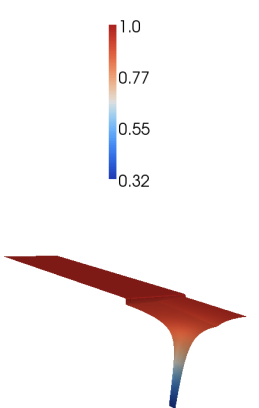

$T=100$ years

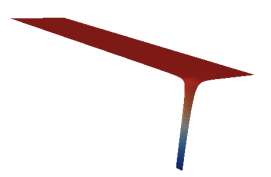

$T=1$ year

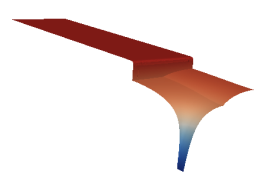

$T=500$ years

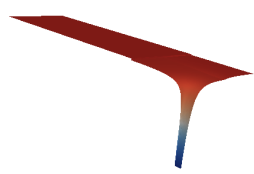

$T=25$ years

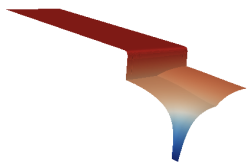

$T=1000$ years

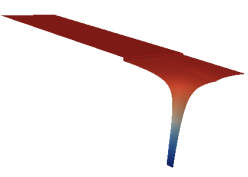

$T=50$ years

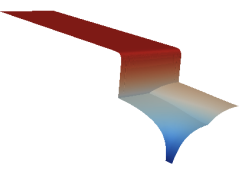

$T=2500$ years

Figure 11: Effective saturation at several times.
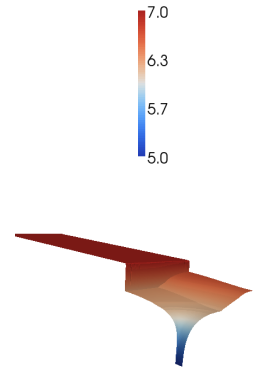

$T=4$ months
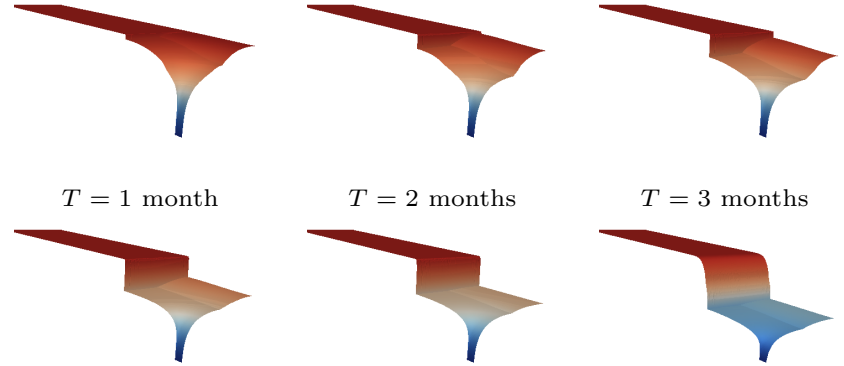

$T=1$ year

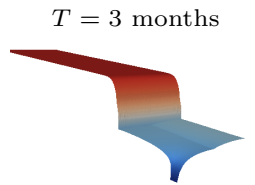

$T=50$ years

Figure 12: Liquid pressure (in $M P a$ ) at several times.

\subsection{A 2D test case: "recovering the natural state"}

The aim of this section is to apply our scheme to a dynamic outflow problem in a fully $2 \mathrm{D}$ domain.

About the domain geometry, we refer to Figure 9. The domain is composed by the following parts : 
- A inner right cylinder, which is composed of two zones (whose depths are $L_{1}$ and $L_{2}$, with $L=L_{1}+L_{2}$ ) having different values of permeability and porosity, i.e. $K_{1}, \phi_{1}$ and $K_{2}, \phi_{2}$. The radius of such a cylinder is $R_{\text {int }}$.

- An external cylindrical ring, whose permeability is much smaller than the previous ones. The external radius of this cylinder is $R_{e x t}$.

- On the top of the domain (i.e. at $x=0$ ) close to the origin $r=0$ there is a portion of the boundary representing the zone from which the fluid can exit. A specific Dirichlet boundary condition will be defined.

The fluid parameters are the same as in the second test case (see Section 3.4 and [41]). Furthermore we consider the presence of capillarity effects (see Table 5 with $\left.P_{r}=1.26 \times 10^{6} \mathrm{~Pa},[41]\right)$.

Concerning the initial and boundary conditions we set :

- No flux conditions on all the boundaries except on the lateral boundary $\left(r=R_{e x t}\right)$ and on the top boundary $\left(x=0,0 \leqslant r \leqslant R_{0}\right)$. On the lateral boundary we impose the Dirichlet condition $X=970 \mathrm{~kg} \cdot \mathrm{m}^{-3}$ and $S=1$. On the top boundary $\left(x=0,0 \leqslant r \leqslant R_{0}\right)$, we impose the Dirichlet condition $X=336.43 \mathrm{~kg} . \mathrm{m}^{-3}$ and $S=0.329$.

- As initial datum, we consider the porous media initially saturated and $X=970 \mathrm{~kg} \cdot \mathrm{m}^{-3}$.

We refer to the Table 8 for the geometry and medium parameters.

In the Fig.10 the time evolution of effective saturation and the liquid pressure is depicted at the middle point of the upper domain, the middle point of the lower cylinder and the point $(100,-500) m$, which is a point under the zone from which the fluid can exit from the domain. Figures 11 and 12 show the saturation and the liquid pressure at several times.

The simulations refer to a simple case modeling "degassing" of a natural basin. The result show that the saturation reduction is more important close to the basin exit, which is expected from physical point of view.

\section{Conclusions}

In this paper we have presented an original approach for analyzing the flow within low porosity porous media of water, which can be in the liquid and in the vapor state. The study has been performed considering isothermal conditions. The aim of the paper is to simulate liquid/vapor system using only one PDE.

We have described the state of system introducing a single variable $X$, representing the global liquid-vapor mixture density. The evolution of $X$ is governed by a PDE which, according to the importance of capillary phenomena, can take different structures. Indeed, in case of non-negligible capillary effects, the equation turns out to be a parabolic, which degenerates to a hyperbolic equation when capillarity is ignored. The flow has been modeled by means of Darcy's 
law, the vapor as a perfect gas and the liquid as a mechanically compressible fluid.

We have performed numerical simulations considering different domains and different sets of physical parameters.

We have performed also simulations considering different values of the liquid mechanical compressibility factor, with the aim of showing that it has little influence on the flow dynamics.

\section{References}

[1] A. Abadpour and M. Panfilov, Method of negative saturations for two-phase compositional flow with oversaturated zones. Transport in Porous Media, v. 79, 2009, p. 197-214.

[2] H. W. Alt and E. DiBenedetto, Nonsteady flow of water and oil through inhomogeneous porous media. Ann. Scuola Norm. Sup. Pisa Cl. Sci. (4) 12 (1985), no. 3, 335-392.

[3] K. Ammar, Degenerate triply nonlinear problems with nonhomogeneous boundary conditions. Cent. Eur. J. Math., 8:548- 568, 2010.

[4] K. Ammar, P.Wittbold and J. Carrillo, Scalar conservation laws with general boundary condition and continuous flux function. J. Differential Equations, 228:11- 139, 2006.

[5] B. Andreianov and K. Sbihi, Scalar conservation laws with nonlinear boundary conditions. C. R. Acad. Sci. Paris, Ser. I, 345:431- 434, 2007

[6] T. Arbogast, M. F. Wheeler, and N. Y. Zhang, A nonlinear mixed finite element method for a degenerate parabolic equation arising in flow in porous media, SIAM J. Numer. Anal., 33 (1996), pp. 1669-1687.

[7] J. Bear and Y. Bachmat, Introduction to Modeling of Transport Phenomena in Porous Media, Theory and Applications of Transport in Porous Media, Vol. 4, Bear J., Ed., Kluwer, 1991.

[8] J. Bear, Dynamics of Fluids in Porous Media, American Elsevier, 1972.

[9] A. Bourgeat, M. Jurak and F. Smaï, Two-phase, partially miscible flow and transport modeling in porous media; application to gas migration in a nuclear waste repository, Comput. Geosci., Vol. 13 (2009), 29-42.

[10] R.H. Brooks and A.T. Corey, Hydraulic porperties of porous media. Colo St Univ Hydrol Pap. 1964;3:1-27.

[11] R. Bürger, H. Frid, and K.H. Karlsen, On the well-posedness of entropy solutions to conservation laws with a zero-flux boundary conditions. J. Math. Anal. Appl., 326:108- 120, 2007. 
[12] F. Bouchut and B. Perthame, Kruzkov's estimates for scalar conservation laws revisited. Trans. Amer. Math. Soc., 350(7):2847-2870, 1998.

[13] J. Carrillo, Entropy Solutions for Nonlinear Degenerate Problems, Arch. Rational Mech. anal., Vol. 147 (1999), p. 269-361.

[14] G. Chavent and J. Jaffré, Mathematical models and finite elements for reservoir simulation. Single phase, multiphase and multicomponent flows through porous media, North-Holland, 1986.

[15] Comsol Multiphysics, http://www.comsol.fr/ .

[16] O. Coussy, Mechanics of porous continua, John Wiley \& Sons (1995).

[17] C. Ebmeyer. Regularity in Sobolev spaces for the fast diffusion and the porous medium equation, J. Math. Anal. Appl., 307(1):134-152, 2005.

[18] R. Eymard, D. Hilhorst and M. Vohralík, A combined finite volume nonconforming/mixed-hybrid finite element scheme for degenerate parabolic problems, Numer. Math. 105(1): 73-131, 2006.

[19] R. Eymard, T. Gallouët, R. Herbin, and A. Michel, Convergence of finite volume schemes for parabolic degenerate equations. Numer. Math., 92:41$82,2002$.

[20] C.R. Faust and J. W. Mercer, Geothermal reservoir simulation - 1. Mathematical models for liquid and vapour dominated hydrothermal systems, Water Res. Research, 15, 23-30 (1979).

[21] C. Galusinski and M. Saad, On a degenerate parabolic system for compressible, immiscible, two-phase flows in porous media. Adv. Differential Equations 9 (2004), no. 11-12, 1235-1278.

[22] W. Jäger and J. Kačur. Solution of doubly nonlinear and degenerate parabolic problems by relaxation schemes, RAIRO Model. Math. Anal. Numer., 29(5):605-627, 1995.

[23] M. T. van Genuchten, A closed-form equation for predicting the hydraulic conductivity of unsaturated soils, Soil Sci. Soc. Am. J., 44, 892-898, 1980. integration, SIAM J. Numer. Anal., 25 (1988), pp. 784-814.

[24] X. Jiang and R. H. Nochetto, Effect of numerical integration for elliptic obstacle problems. Numer. Math., Vol. 67 (1994), no. 4, 501-512.

[25] C. Johnson, Error estimates and adaptive time-step control for a class of one-step methods for stiff ordinary differential equations, SIAM J. Numer. Anal., Vol. 25, No.4, 1988, 908-926.

[26] D. Kondepudi and I. Prigogine, Modern Thermodynamics, John Wiley and Sons, p.167, 1998. 
[27] D. Kröner and S. Luckhaus, Flow of oil and water in a porous medium. J. Differential Equations 55 (1984), no. 2, 276-288.

[28] C. Mascia, A. Porretta, and A. Terracina, Nonhomogeneous Dirichlet problems for degenerate parabolic-hyperbolic equations. Arch. Ration. Mech. Anal., 163(2):87- 124, 2002.

[29] A. Michel and J. Vovelle. Entropy formulation for parabolic degenerate equations with general Dirichlet boundary conditions and application to the convergence of FV methods. SIAM J. Numer. Anal., 41(6):2262- 2293 (electronic), 2003.

[30] A. Mikelić, An existence result for the equations describing a gas-liquid two-phase flow, Comptes rendus Mécanique, Volume 337, Issue 4, 2009, p. 226-232.

[31] Modular Modeling, http://mmodd.univ-lyon1.fr/projects/mmodd/.

[32] Y. Mualem, A new model for predicting the hydraulic conductivity of unsaturated porous media, Water Resour. Res., 12, 513-522, 1976.

[33] R. H. Nochetto and C. Verdi, Approximation of degenerate parabolic problems using numerical integration, SIAM J. Numer. Anal., 25 (1988), pp. $784-814$

[34] R. H. Nochetto, A note on the approximation of free boundaries by finite element methods, RAIRO Model. Math. Anal. Numer., 20 (1986), pp 355368.

[35] M. Panfilov, and M. Rassoulzadeh. Interfaces of phase transition and method of negative saturations for compositional flow with diffusion and capillarity in porous media. Transport in Porous Media, 83(1):73- 98, 2010.

[36] I.S. Pop. Error estimates for a time discretization method for the Richard's equation, Comput. Geosci., 6(2):141-160, 2002.

[37] I.S. Pop and W.A. Yong. A numerical approach to degenerate parabolic equations. Numer. Math., 92(2):357-381, 2002.

[38] F.Radu, I. S. Pop and P. Knabner. Order of convergence estimates for an Euler implicit, mixed finite element discretization of Richards equation. SIAM J. Numer. Anal., 42 (2004), pp. 1452-1478.

[39] I. S. Pop and B. Schweizer. Regularization schemes for degenerate Richards equations and outflow conditions, Mathematical Models and Methods in Applied Sciences (M3AS), 2011, Isuue 1, pp. 1-26.

[40] K.R. Rajagopal and L. Tao, Mechanics of Mixtures, World Scientific, Singapore, 1995. 
[41] G. G. Tsypkin and A. W. Woods, Vapor extraction from a water-saturated geothermal reservoir, J. Fluid Mech., 506, 315-330 (2004).

[42] G. G. Tsypkin, On water-steam phase transition front in geothermal reservoirs USA, Proc. Stanford Geotherm. Workshop 22, 359-367 (1997). 